\title{
Effect of opioid-substitution therapy and mental health counseling on HIV risk among hepatitis $\mathrm{C}$-infected individuals
}

This article was published in the following Dove Press journal:

Clinical Epidemiology

Zahid A Butt,' Nabin Shrestha,' Dionne Gesink, ${ }^{2}$ Michelle Murti, ${ }^{2,3}$ Jane A Buxton, ${ }^{1,4}$ Mark Gilbert, ${ }^{4}$ Robert F Balshaw, ${ }^{4}$ Stanley Wong, ${ }^{4}$ Margot Kuo, ${ }^{4}$ Jason Wong, ${ }^{1,4}$ Amanda Yu, ${ }^{4}$ Maria Alvarez, ${ }^{4}$ Hasina Samji, ${ }^{4}$ David Roth, ${ }^{4}$ Theodora Consolacion, ${ }^{4}$ Mark W Hull,, ${ }^{5,6}$ Gina Ogilvie, ${ }^{1,4}$ Mark W Tyndall, 1,4 Mel Krajden, ${ }^{1,4,7}$ Naveed Z Janjua ${ }^{1,4}$

'School of Population and Public Health, University of British Columbia, Vancouver BC, ${ }^{2}$ Dalla Lana School of Public Health, University of Toronto, ${ }^{3}$ Public Health Ontario, Toronto, ON, ${ }^{4} \mathrm{Clinical}$ Prevention Services, British Columbia Centre for Disease Control, ${ }^{5}$ Division of AIDS, Faculty of Medicine, University of British Columbia, ${ }^{6}$ AIDS Research Program, British Columbia Centre for Excellence in HIV/AIDS, ${ }^{7}$ BCCDC Public Health Laboratory, Vancouver, BC, Canada

\section{Video abstract}

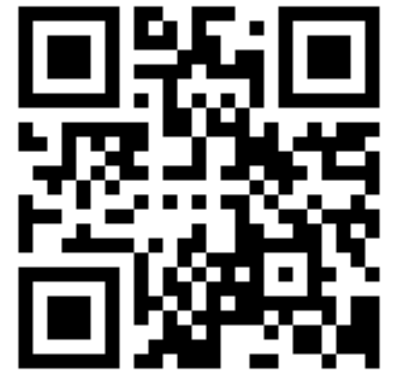

Point your SmartPhone at the code above. If you have a QR code reader the video abstract will appear. Or use: https://www.youtube.com/watch?y=AyezP TwatQ

Correspondence: Zahid A Butt School of Population and Public Health University of British Columbia, 2206 East Mall, Vancouver, BC V6T IZ3, Canada Tel +l 6047072485

Fax +I 6047072690

Email zahid.butt@ubc.ca
Background: Understanding differences in HIV incidence among people living with hepatitis $\mathrm{C}$ virus (HCV) can help inform strategies to prevent HIV infection. We estimated the time to HIV diagnosis among HCV-positive individuals and evaluated factors that could affect HIVinfection risk in this population.

Patients and methods: The British Columbia Hepatitis Testers Cohort includes all BC residents ( $\sim 1.5$ million: about a third of all residents) tested for HCV and HIV from 1990 to 2013 and is linked to administrative health care and mortality data. All HCV-positive and HIV-negative individuals were followed to measure time to HIV acquisition (positive test) and identify factors associated with HIV acquisition. Adjusted HRs (aHRs) were estimated using Cox proportional-hazard regression.

Results: Of 36,077 HCV-positive individuals, 2,169 (6\%) acquired HIV over 266,883 years of follow-up (overall incidence of 8.1 per 1,000 person years). Overall median (IQR) time to HIV infection was 3.87 (6.06) years. In Cox regression, injection-drug use (aHR 1.47, 95\% CI 1.33-1.63), HBV infection (aHR 1.34, 95\% CI 1.16-1.55), and being a man who has sex with men (aHR 2.78, 95\% CI 2.14-3.61) were associated with higher risk of HIV infection. Opioidsubstitution therapy (OST) (aHR 0.59, 95\% CI 0.52-0.67) and mental health counseling (aHR $0.48,95 \%$ CI $0.43-0.53$ ) were associated with lower risk of HIV infection.

Conclusion: Injection-drug use, HBV coinfection, and being a man who has sex with men were associated with increased HIV risk and engagement in OST and mental health counseling were associated with reduced HIV risk among HCV-positive individuals. Improving access to OST and mental health services could prevent transmission of HIV and other blood-borne infections, especially in settings where access is limited.

Keywords: opioid-substitution therapy, mental health counseling, HIV, risk, hepatitis

\section{Introduction}

Hepatitis $\mathrm{C}$ virus (HCV) infection and HIV infection commonly co-occur because of shared transmission routes and risk behaviors. Globally, 2.7 million people have HIV-HCV coinfection, and of these $90 \%$ are among people who inject drugs (PWID). ${ }^{1} \mathrm{HIV}$ infection among $\mathrm{HCV}^{+}$individuals is a major predictor of morbidity ${ }^{2,3}$ and mortality. ${ }^{4,5}$ Highly effective, well-tolerated, short-course, direct-acting antiviral agents with cure rates approaching 95\% have revolutionized the treatment of HCV. These agents could prevent progressive liver disease and hence reduce morbidity and mortality. Prevention of HIV infection by addressing risk factors among people living with $\mathrm{HCV}$ or treated for $\mathrm{HCV}$ but potentially at risk of HIV could further reduce morbidity and mortality. 
Risk factors for $\mathrm{HCV}$ coinfection among people living with HIV include injection-drug use (IDU), ${ }^{6}$ alcohol use, ${ }^{6,7}$ unsafe sex among gay, bisexual, and other men who have sex with men (MSM), ${ }^{8,9}$ and social disparities. ${ }^{10}$ Potential preventive factors for HIV transmission, particularly among PWID, include opioid-substitution therapy (OST), ${ }^{11,12}$ mental health counseling, and other harm-reduction strategies. ${ }^{12,13}$ OST and mental health counseling have also been observed to reduce HCV reinfection among PWID. ${ }^{14}$ However, data on risk factors for HIV among people living with HCV are limited, and the role of preventive factors has not been investigated. Coinfected individuals have multiple co-occurring infections and comorbidities; therefore, data on preventive factors for HIV infection from a real-world setting will inform programs that could reduce the burden of HIV among HCV-infected individuals. In this paper, we present an analysis of a large population-based cohort to estimate the risk of HIV and factors preventing and promoting this risk among people living with HCV in British Columbia.

\section{Patients and methods}

\section{Study cohort}

The British Columbia Hepatitis Testers Cohort (BC-HTC) includes all individuals ( $\sim 1.5$ million; approximately a third of all $\mathrm{BC}$ residents) tested for $\mathrm{HCV}$ or $\mathrm{HIV}$ at the $\mathrm{BC}$ Centre for Disease Control Public Health Laboratory (BCCDCPHL) or diagnosed with HCV, HBV, HIV/AIDS, or active tuberculosis (TB). This cohort is linked with data on medical visits, hospitalizations, prescription drugs, cancers, and deaths (Tables S1 and S2). Almost all HIV and HCV testing in $\mathrm{BC}$ is performed at the BCCDC-PHL. All prescriptions dispensed in $\mathrm{BC}$ are recorded in a centralized system called PharmaNet, irrespective of the payer. Detailed characteristics of the cohort, including linkage and related data, are presented elsewhere. ${ }^{15,16}$ Data linkages were approved by the data stewards of the BCCDC, BCCDC-PHL, BC Ministry of Health, BC Vital Statistics Agency, and BC Cancer Registry. Approval for data linkage with the Ministry of Health data sets was granted under the BCCDC public health mandate to conduct surveillance and program evaluation. Data were deidentified before analyses. ${ }^{15}$ This study was approved by the Behavioral Research Ethics Board (H15-01776) at the University of British Columbia.

\section{Study population}

Individuals diagnosed with HCV during 1990-2013 but negative for HIV were eligible for inclusion in this analysis. Individuals who tested positive for $\mathrm{HCV}$ and negative for HIV were followed for new diagnoses of HIV. A person's time at risk started at $\mathrm{HCV}$ diagnosis and ended at HIV diagnosis, December 31, 2013 (end of follow-up), or death, whichever occurred earlier.

\section{Outcome assessment}

The outcome variable of interest was a time to HIV infection at least 6 weeks after HCV diagnosis. The date of HIV infection was estimated as the midpoint between the last HIVnegative test and the first $\mathrm{HIV}^{+}$test. ${ }^{11} \mathrm{All} \mathrm{HCV}^{+}$individuals who tested positive for HIV on the same day or within 6 weeks of HCV diagnosis were excluded from the analysis.

\section{Definitions}

An individual testing positive for $\mathrm{HCV}$ antibodies, HCV RNA or genotype, or who had been reported as a case of HCV to public health was considered an "HCV case". ${ }^{15}$ Participants who were $\mathrm{HCV}$-antibody ${ }^{+}$at their first test on record were considered to have prevalent infection $\left(\mathrm{HCV}^{+}\right.$-prevalent), while individuals with a negative test followed by a positive test were considered seroconverters $\left(\mathrm{HCV}^{+}\right.$seroconverters). ${ }^{16}$ An individual included in provincial HIV/AIDS-surveillance data or who had a positive HIV laboratory test result was considered a case based on provincial HIV laboratorytest-interpretation guidelines. Additional HIV cases were ascertained through a validated algorithm requiring two medical visits or a hospitalization. ${ }^{17} \mathrm{HBV}$ and active TB diagnoses were based on provincial and national guidelines. Socioeconomic status was assessed using the Québec Index of Material and Social Deprivation, which is based on Canadian census data on small-area units. ${ }^{18}$ The material component of the index includes indicators for education, employment, and income, whereas the social component comprises indicators related to marital status and family structure. Assessment of IDU, major mental illness, and problematic alcohol use was based on diagnostic codes for medical visits and hospitalizations in respective databases (Table S2). OST was based on prescriptions recorded in PharmaNet. IDU, problematic alcohol use, OST, and mental illnesses were evaluated across all data within the interval between HCV and HIV diagnosis and within 3 years of the first positive or last negative HIV or HCV test. Additionally, OST was evaluated as a time-varying covariate for this analysis. An individual was considered off OST if the interval between two successive prescriptions for OST was $>28$ days. ${ }^{19}$ Mental health counseling was defined as any mental healthcounseling visit during the follow-up period and was based on fee-item codes from a Medical Services Plan (medical 
visits; Table S2). Mental health counseling includes services provided by a psychiatrist and comprises individual, family, and group therapy and telehealth services.

Data on sexual orientation were not available for all $\mathrm{HCV}^{+}$ individuals. We developed and validated an algorithm for identification of MSM status in a subset of data $(n=5,382)$ on which sexual orientation variable was available. A model was developed to predict MSM status using penalized generalized linear model with testing patterns for HIV and sexually transmitted infections (STIs), previous STI diagnoses, substance use, visit to a clinic providing services to gay and other bisexual men, and residence in an area with higher percentage of MSM as predictor variables. The selected model had an area under the receiver-operating curve of $0.78 \%$, $91.8 \%$ specificity, and $42.0 \%$ sensitivity.

\section{Statistical analysis}

Characteristics of $\mathrm{HCV}^{+}$-prevalent and $\mathrm{HCV}^{+}$seroconverters who were followed until HIV seroconversion were described separately. This stratification was done because our previous research showed differences in characteristics between these two populations. ${ }^{16} \mathrm{HCV}^{+}$-prevalent and $\mathrm{HCV}^{+}$seroconverters are different in terms of demographic and risk factors. $\mathrm{HCV}^{+}$seroconverters reflect ongoing risk activities, including IDU, while people with prevalent infection may not be engaged in high-risk activities, as shown in our previous work. HIV-HBV coinfection, major mental illness, illicitdrug use, problematic alcohol use, and socioeconomic deprivation are higher among $\mathrm{HCV}^{+}$seroconverters than $\mathrm{HCV}^{+}$-prevalent individuals. ${ }^{16}$ Therefore, transmission risk of HIV may be different in each of these groups, which were selected a priori for stratified analysis to account for differing baseline risk profiles of people. Incidence rates of HIV among $\mathrm{HCV}^{+}$individuals (all, $\mathrm{HCV}^{+}$-prevalent, and $\mathrm{HCV}^{+}$ seroconverters) were calculated by dividing the number with HIV by person-years at risk. Cumulative-incidence curves were constructed comparing HIV incidence among various groups. Cox proportional-hazard regression analysis was conducted to assess the association of factors with HIV risk, and results summarized using HRs with corresponding 95\% CIs. Analyses were performed in SAS/STAT software version 9.4 and $\mathrm{R}$ version 3.3.2.

\section{Results}

A total of $36,077 \mathrm{HCV}^{+}$individuals were eligible for inclusion in our study (Figure 1). Of these, 33,908 (93.9\%) had not acquired HIV infection and 2,169 (6.0\%) had developed HIV infection during 266,883 person years of follow-up.
Among HCV-infected individuals, 30,614 (84.8\%) were $\mathrm{HCV}^{+}$-prevalent whereas 5,463 (15.1\%) were $\mathrm{HCV}^{+}$seroconverters (Table 1). A similar proportion of HIV cases were detected among $\mathrm{HCV}^{+}$seroconverters $(6.1 \%, 334$ of 5,463$)$ and $\mathrm{HCV}^{+}$-prevalent individuals $(5.9 \%, 1,835$ of 30,614$)$.

\section{Distribution of characteristics across HIV and HCV groups}

The majority of HCV-infected individuals were male: $66.6 \%$ of $\mathrm{HCV}^{+}$-prevalent individuals and $53.5 \%$ of $\mathrm{HCV}^{+}$seroconverters. Most of the $\mathrm{HCV}^{+}$-prevalent individuals were born during 1945-1964 (66.9\%) and diagnosed between 35 and 54 years of age, whereas the majority of $\mathrm{HCV}^{+}$seroconverters were born after 1964 (73.9\%) and diagnosed between the ages of 25 and 44 years (Table 1). The majority of $\mathrm{HCV}^{+}$-prevalent individuals (86.8\%) and $\mathrm{HCV}^{+}$seroconverters (89.1\%) lived in urban areas. The majority of $\mathrm{HCV}^{+}$individuals were materially and socially deprived; however, $\mathrm{HCV}^{+}$seroconverters were more socially ( $45.5 \%$ vs $38.7 \%$ : fifth quintile) and materially (32.5\% vs 30.4\%: fifth quintile) deprived than $\mathrm{HCV}^{+}$-prevalent individuals. A higher proportion of $\mathrm{HCV}^{+}$ seroconverters had a history of illicit drug use $(57.9 \%$ vs $23.7 \%$ ), IDU (40.5\% vs $15.1 \%$ ), and problematic alcoholic use $(17.6 \%$ vs $10.4 \%)$ compared to $\mathrm{HCV}^{+}$-prevalent individuals. Similarly, compared to $\mathrm{HCV}^{+}$-prevalent individuals, $\mathrm{HCV}^{+}$seroconverters had a higher proportion of major mental illnesses (22.9\% vs 9.9\%), depression (40.7\% vs $23.5 \%$ ), and psychosis (6.8\% vs $2.7 \%)$. HBV was more commonly diagnosed in $\mathrm{HCV}^{+}$-prevalent individuals $(5.6 \%)$ than $\mathrm{HCV}^{+}$ seroconverters (3.7\%; Table 1). A similar trend was observed for these factors within 3 years of the first positive or last negative HIV or HCV test (Table 1).

\section{Incidence of HIV infection by HCV group}

The overall HIV-incidence rate among $\mathrm{HCV}^{+}$individuals was $8.1 / 1,000$ person-years for a total of 266,883 person-years of follow-up. The $\mathrm{HIV}$-incidence rate among $\mathrm{HCV}^{+}$seroconverters was higher (10.2/1,000 person-years) compared to those with prevalent $\mathrm{HCV}$ infection (7.8/1,000 person-years) at diagnosis. Overall median (interquartile range [IQR]) time to HIV infection was 3.87 (6.06) years, shorter for seroconverters (3.63) years than prevalent HCV infections (3.95 years; Table 2). Being male, having a history of IDU, mental illness, $\mathrm{HBV}$, MSM status, younger age, and urban residence were associated with a higher incidence rate of HIV; however, the magnitude was lower among $\mathrm{HCV}^{+}$-prevalent individuals than $\mathrm{HCV}^{+}$seroconverters. People on OST and receiving mental 


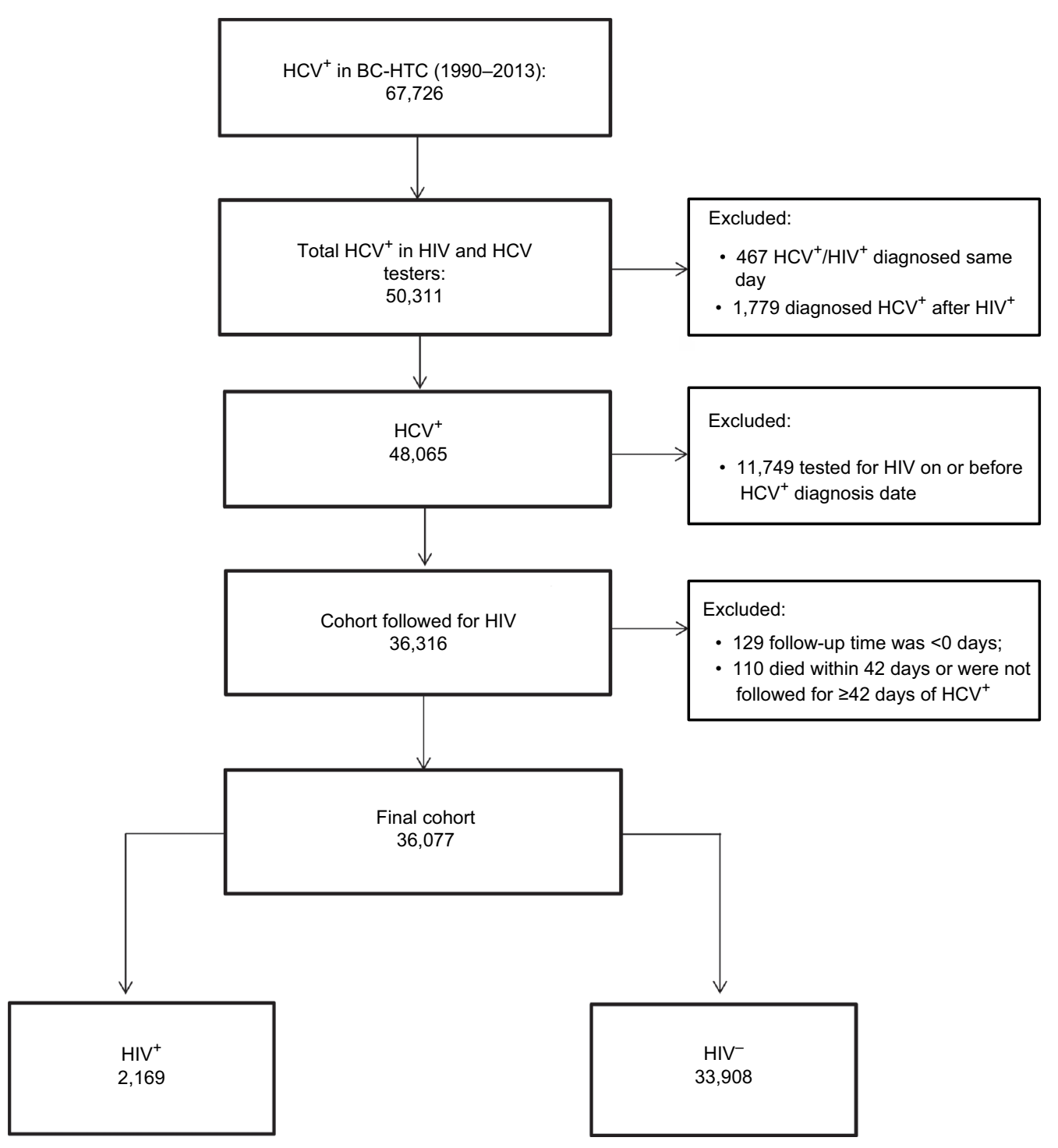

Figure I Selection of participants for HIV infection analysis in British Columbia, Canada. Abbreviations: HCV, hepatitis C virus; BC-HTC, British Columbia Hepatitis Testers Cohort.

health counseling had lower HIV incidence for both $\mathrm{HCV}^{+}$ seroconverters and $\mathrm{HCV}^{+}$-prevalent individuals (Table 2).

The cumulative incidence of HIV increased with time for both IDU and non-IDU, but the increase was steeper for IDU $(P<0.0001$; Figure 2). Cumulative incidence of HIV increased with time in MSM, but was similar between females and males who did not have sex with men (Figure 2). For individuals who were on OST, the cumulative incidence of HIV was much lower than those who were not on OST and decreased with time (Figure 2). The cumulative incidence of HIV increased with time among those who received mental health counseling, as well as among those who did not receive mental health counseling, but the increase was more pronounced in those who did not receive mental health counseling (Figure 2).

\section{Factors associated with risk of HIV infection among HCV-infected individuals} In the multivariable Cox proportional-hazard model for all HCV diagnoses, MSM status (adjusted HR 2.78, 95\% CI 2.14-3.61), urban residence (adjusted HR 1.45, 95\% CI 1.22-1.72), IDU (adjusted HR 1.47, 95\% CI 1.33-1.63), HBV (adjusted HR 1.34, 95\% CI 1.16-1.55), and lower age at $\mathrm{HCV}$ diagnosis were significantly associated with increased risk of HIV infection. Conversely, being on OST (adjusted HR $0.59,95 \%$ CI 0.52-0.67) and having received mental 
Table I Characteristics of HCV-diagnosed persons followed until time to HIV infection in the BC Hepatitis Testers Cohort

\begin{tabular}{|c|c|c|c|}
\hline \multirow[t]{2}{*}{ Variables } & \multirow{2}{*}{$\begin{array}{l}\mathrm{HCV}^{+} \text {seroconverters, } \mathrm{n}(\%) \\
5,463(15 . \mathrm{I})\end{array}$} & \multirow{2}{*}{$\begin{array}{l}\text { HCV+-prevalent, n (\%) } \\
30,614(84.8)\end{array}$} & \multirow{2}{*}{$\begin{array}{c}\text { Total, n (\%) } \\
36,077(100)\end{array}$} \\
\hline & & & \\
\hline \multicolumn{4}{|l|}{ Sex } \\
\hline Female & $2,540(46.5)$ & $10,236(33.4)$ & $12,776(35.4)$ \\
\hline Male & $2,923(53.5)$ & $20,378(66.6)$ & $23,301(64.6)$ \\
\hline \multicolumn{4}{|c|}{ Sex and sexual orientation } \\
\hline Male, not MSM & $2,866(52.5)$ & $20,017(65.4)$ & $22,883(63.4)$ \\
\hline Male, yes MSM & $57(1.0)$ & $361(1.2)$ & $418(1.2)$ \\
\hline Female & $2,540(46.5)$ & $10,236(33.4)$ & $12,776(35.4)$ \\
\hline \multicolumn{4}{|l|}{ Birth year } \\
\hline Before 1945 & $43(0.8)$ & $\mathrm{I}, 7 \mathrm{I} 5(5.6)$ & $\mathrm{I}, 758(4.9)$ \\
\hline $1945-1964$ & $\mathrm{I}, 379(25.2)$ & $20,489(66.9)$ & $21,868(60.6)$ \\
\hline After 1964 & $4,04 \mid(73.9)$ & $8,410(27.5)$ & $|2,45|(34.5)$ \\
\hline \multicolumn{4}{|c|}{ Age at HCV diagnosis } \\
\hline$<15$ years & $9(0.2)$ & $146(0.5)$ & $155(0.4)$ \\
\hline 15-24 years & I, $193(21.8)$ & $1,790(5.9)$ & $2,983(8.3)$ \\
\hline $25-34$ years & $2,200(40.3)$ & $7,164(23.4)$ & $9,364(26)$ \\
\hline $35-44$ years & $1,438(26.3)$ & $11,376(37.2)$ & $12,8 \mid 4(35.5)$ \\
\hline $45-54$ years & $490(9)$ & $7,369(24)$ & $7,859(21.8)$ \\
\hline$>54$ years & $133(2.4)$ & $2,769(9)$ & $2,902(8)$ \\
\hline \multicolumn{4}{|c|}{ Year of HCV diagnosis } \\
\hline Before 2000 & $\mathrm{I}, \mathrm{I} 05(20.2)$ & $17,222(56.3)$ & I8,327 (50.8) \\
\hline 2000-2004 & $1926(35.3)$ & $7,236(23.6)$ & $9,162(25.4)$ \\
\hline 2005-2008 & $\mathrm{I}, 4 \mathrm{I} 5(25.9)$ & $3,644(11.9)$ & $5,059(14.0)$ \\
\hline $2009-2011$ & $730(13.4)$ & I,77I (5.8) & $2,501(6.9)$ \\
\hline After $201 \mathrm{I}$ & $287(5.2)$ & $74 I(2.4)$ & $1,028(2.8)$ \\
\hline \multicolumn{4}{|l|}{ Urban } \\
\hline Unknown & $85(1.6)$ & $674(2.2)$ & $759(2.1)$ \\
\hline No & $507(9.3)$ & $3,363(10.9)$ & $3,870(10.7)$ \\
\hline Yes & $4,87 \mid(89.1)$ & $26,577(86.8)$ & $31,448(87.2)$ \\
\hline \multicolumn{4}{|c|}{ Social deprivation quintile at time of test } \\
\hline Unknown & $\mathrm{III}(2)$ & $657(2.2)$ & $768(2.1)$ \\
\hline QI (most privileged) & $433(7.9)$ & $2,876(9.4)$ & $3,309(9.2)$ \\
\hline Q2 & $535(9.8)$ & $3,747(12.2)$ & $4,282(11.9)$ \\
\hline Q3 & $800(14.6)$ & $5,099(16.6)$ & $5,899(16.3)$ \\
\hline Q4 & $1,100(20.1)$ & 6,399 (20.9) & $7,499(20.8)$ \\
\hline Q5 (most deprived) & $2,484(45.5)$ & $11,836(38.7)$ & $14,320(39.7)$ \\
\hline \multicolumn{4}{|c|}{ Material deprivation quintile at time of test } \\
\hline Unknown & III (2) & $657(2.2)$ & $768(2.1)$ \\
\hline QI (most privileged) & $641(11.7)$ & $3,728(12.2)$ & $4,369(12.1)$ \\
\hline Q2 & $751(13.7)$ & $4,635(15.1)$ & $5,386(14.9)$ \\
\hline Q3 & $869(15.9)$ & $5,347(17.4)$ & $6,216(17.2)$ \\
\hline Q4 & $1,315(24.1)$ & $6,944(22.7)$ & $8,259(22.9)$ \\
\hline Q5 (most deprived) & $1,776(32.5)$ & $9,303(30.4)$ & II,079 (30.7) \\
\hline \multicolumn{4}{|l|}{ Illicit-drug use $\mathrm{a}^{\mathrm{a}}$} \\
\hline No & $2,298(42.1)$ & $23,352(76.3)$ & $25,650(7 \mathrm{I} .1)$ \\
\hline Yes & $3,165(57.9)$ & $7,262(23.7)$ & $10,427(28.9)$ \\
\hline \multicolumn{4}{|l|}{ IDUa } \\
\hline No & $3,249(59.5)$ & $26,000(84.9)$ & $29,249(8 I . I)$ \\
\hline Yes & $2,214(40.5)$ & $4,614(15.1)$ & $6,828(18.9)$ \\
\hline \multicolumn{4}{|l|}{ OST $^{\mathbf{a}}$} \\
\hline No & $4,079(74.7)$ & $27,903(91.1)$ & $31,982(88.6)$ \\
\hline Yes & I,384 (25.3) & $2,711(8.9)$ & 4,095 (II.4) \\
\hline \multicolumn{4}{|c|}{ Major mental illness ${ }^{a}$} \\
\hline No & $4,213(77.1)$ & $27,595(90.1)$ & $31,808(88.2)$ \\
\hline Yes & $1,250(22.9)$ & $3,019(9.9)$ & 4,269 (II.8) \\
\hline
\end{tabular}


Table I (Continued)

\begin{tabular}{|c|c|c|c|}
\hline \multirow[t]{2}{*}{ Variables } & \multirow{2}{*}{$\begin{array}{l}\mathrm{HCV}^{+} \text {seroconverters, } \mathrm{n}(\%) \\
5,463(15.1)\end{array}$} & \multirow{2}{*}{$\begin{array}{l}\text { HCV+-prevalent, n (\%) } \\
30,614(84.8)\end{array}$} & \multirow{2}{*}{$\begin{array}{l}\text { Total, n (\%) } \\
36,077(100) \\
\end{array}$} \\
\hline & & & \\
\hline \multicolumn{4}{|c|}{ Depression $^{a}$} \\
\hline No & $3,238(59.3)$ & $23,426(76.5)$ & $26,664(73.9)$ \\
\hline Yes & $2,225(40.7)$ & $7,188(23.5)$ & $9,413(26.1)$ \\
\hline \multicolumn{4}{|l|}{ Psychosis $^{a}$} \\
\hline No & $5,091(93.2)$ & $29,790(97.3)$ & $34,881(96.7)$ \\
\hline Yes & $372(6.8)$ & $824(2.7)$ & $1,196(3.3)$ \\
\hline \multicolumn{4}{|c|}{ Problematic alcohol use $^{a}$} \\
\hline No & $4,502(82.4)$ & $27,418(89.6)$ & $31,920(88.5)$ \\
\hline Yes & $961(17.6)$ & $3,196(10.4)$ & $4,157(11.5)$ \\
\hline \multicolumn{4}{|l|}{ Active TB ${ }^{a}$} \\
\hline No & $5,458(99.9)$ & 30,585 (99.9) & 36,043 (99.9) \\
\hline Yes & $5(0.1)$ & $29(0.1)$ & $34(0.1)$ \\
\hline \multicolumn{4}{|c|}{ Hepatitis $\mathbf{B}^{\mathbf{a}}$} \\
\hline No & $5,368(98.3)$ & $30,311(99.0)$ & 35,679 (98.9) \\
\hline Yes & $95(1.7)$ & $303(1.0)$ & $398(1.1)$ \\
\hline \multicolumn{4}{|c|}{ Illicit-drug use ${ }^{b}$} \\
\hline No & $\mathrm{I}, 6 \mathrm{I} 4(29.5)$ & I6,884 (55.I) & I8,498 (5।.3) \\
\hline Yes & $3,849(70.5)$ & I3,730 (44.8) & $17,579(48.7)$ \\
\hline \multicolumn{4}{|l|}{ IDU } \\
\hline No & $2,561(46.9)$ & $20,626(67.3)$ & $23,187(64.3)$ \\
\hline Yes & $2,902(53.1)$ & $9,988(32.6)$ & $12,890(35.7)$ \\
\hline \multicolumn{4}{|l|}{ OST $^{b}$} \\
\hline No & $2,840(51.9)$ & $22,925(74.9)$ & $25,765(71.4)$ \\
\hline Yes & $2,623(48.0)$ & $7,689(25.1)$ & $10,312(28.6)$ \\
\hline \multicolumn{4}{|c|}{ Major mental illness ${ }^{b}$} \\
\hline No & $3,767(69.0)$ & $23,779(77.7)$ & $27,546(76.3)$ \\
\hline Yes & $1,696(31.0)$ & $6,835(22.3)$ & $8,531(23.6)$ \\
\hline \multicolumn{4}{|c|}{ Depression ${ }^{b}$} \\
\hline No & $2,647(48.4)$ & I7,049 (55.7) & $19,696(54.6)$ \\
\hline Yes & $2,816(51.5)$ & I3,565 (44.3) & $|6,38|(45.4)$ \\
\hline \multicolumn{4}{|l|}{ Psychosis $^{b}$} \\
\hline No & $4,84 I(88.6)$ & $28,447(92.9)$ & $33,288(92.3)$ \\
\hline Yes & $622(11.3)$ & $2,167(7.0)$ & $2,789(7.7)$ \\
\hline \multicolumn{4}{|c|}{ Mental health counseling } \\
\hline No & $3,422(62.6)$ & $22,405(73.2)$ & $25,827(71.6)$ \\
\hline Yes & $204 I(37.4)$ & $8,209(26.8)$ & $10,250(28.4)$ \\
\hline \multicolumn{4}{|c|}{ Mental health-counseling visits ${ }^{b}$} \\
\hline 0 & $3,422(62.6)$ & $22,405(73.2)$ & $25,827(7 I .6)$ \\
\hline I & $417(7.6)$ & $1,725(5.6)$ & $2,142(5.9)$ \\
\hline$\geq 2$ & $\mathrm{I}, 624(29.7)$ & $6,484(21.2)$ & $8,108(22.5)$ \\
\hline \multicolumn{4}{|c|}{ Problematic alcohol use $^{b}$} \\
\hline No & $4,176(76.4)$ & $24,065(78.6)$ & $28,24 I(78.3)$ \\
\hline Yes & $1,287(23.5)$ & $6,549(21.4)$ & $7,836(21.7)$ \\
\hline \multicolumn{4}{|l|}{ Active TB ${ }^{b}$} \\
\hline No & $5,452(99.8)$ & $30,497(99.6)$ & $35,949(99.6)$ \\
\hline Yes & $\mathrm{II}(0.20)$ & $117(0.3)$ & $128(0.3)$ \\
\hline \multicolumn{4}{|c|}{ Hepatitis $\mathbf{B}^{\mathrm{b}}$} \\
\hline No & $5,261(96.3)$ & $28,893(94.3)$ & $34,154(94.6)$ \\
\hline Yes & $202(3.7)$ & $|, 72|(5.6)$ & $1923(5.3)$ \\
\hline
\end{tabular}

Note: ${ }^{a}$ Within 3 years of first positive or last negative HIV or HCV test; ${ }^{b}$ risk factor/disease history in interval between HCV and HIV diagnosis.

Abbreviations: $\mathrm{HCV}$, hepatitis C virus; IDU, injection-drug use; MSM, men who have sex with men; OST, opioid-substitution therapy; TB, tuberculosis. 
Table 2 HIV incidence per I,000 person-years by HCV group, BC Hepatitis Testers Cohort, 1990-2013

\begin{tabular}{|c|c|c|c|c|c|c|}
\hline \multirow[t]{2}{*}{ Variables } & \multicolumn{2}{|c|}{ HCV infection } & \multicolumn{2}{|c|}{$\mathrm{HCV}^{+}$seroconverters } & \multicolumn{2}{|c|}{$\mathrm{HCV}^{+}$-prevalent } \\
\hline & $\begin{array}{l}\text { n/person- } \\
\text { years }\end{array}$ & $\begin{array}{l}\text { Incidence } \\
(95 \% \mathrm{Cl})\end{array}$ & $\begin{array}{l}\text { n/person- } \\
\text { years }\end{array}$ & $\begin{array}{l}\text { Incidence } \\
(95 \% \mathrm{Cl})\end{array}$ & $\begin{array}{l}\text { n/person- } \\
\text { years }\end{array}$ & $\begin{array}{l}\text { Incidence } \\
(95 \% \mathrm{Cl})\end{array}$ \\
\hline All HIV cases & $2,169 / 266,883.0$ & $8.1(7.8-8.5)$ & $334 / 32,646.2$ & $10.2(9.2-11.4)$ & I,835/234,236.8 & $7.8(7.5-8.2)$ \\
\hline \multicolumn{7}{|c|}{ Sex and sexual orientation } \\
\hline Female & $723 / 95,668.7$ & $7.6(7.0-8.1)$ & $|57 /| 5,284 . \mid$ & $10.3(8.8-12.0)$ & $566 / 80,384.6$ & $7.0(6.5-7.6)$ \\
\hline Male, not MSM & I,385/I68,363.2 & $8.2(7.8-8.7)$ & $164 / 17,139.1$ & $9.6(8.2-11.2)$ & $|, 22| / \mid 15 I, 224.0$ & $8.1(7.6-8.5)$ \\
\hline Male, yes MSM & $6 \mathrm{I} / 2,85 \mathrm{I} .2$ & $21.4(16.6-27.5)$ & $13 / 223.0$ & $58.3(33.9-100.4)$ & $48 / 2,628.2$ & I8.3 (I3.8-24.2) \\
\hline \multicolumn{7}{|l|}{ IDUa } \\
\hline No & $996 / 145,739.8$ & $6.8(6.4-7.3)$ & $\mid \mathrm{II} / \mathrm{II}, 770.4$ & $9.9(8.3-11.9)$ & $879 / 133,969.4$ & $6.6(6.1-7.0)$ \\
\hline Yes & $\mathrm{I}, 173 / 121,143.2$ & $9.7(9.1-10.3)$ & $217 / 20,875.8$ & $10.4(9.1-11.9)$ & $956 / 100,267.5$ & $9.5(8.9-10.2)$ \\
\hline \multicolumn{7}{|l|}{ Drug misuse $^{a}$} \\
\hline No & $756 / 108,458.6$ & $7.0(6.5-7.5)$ & $86 / 6,419.7$ & $13.4(10.8-16.5)$ & $670 / 102,038.9$ & $6.6(6.1-7.1)$ \\
\hline Yes & I,4I3/I58,424.4 & $8.9(8.5-9.4)$ & $248 / 26,226.5$ & $9.5(8.3-10.7)$ & I,I65/I32,198.0 & $8.8(8.3-9.3)$ \\
\hline \multicolumn{7}{|l|}{ OST $^{a}$} \\
\hline No & I,564/I72,755.8 & $9.1(8.6-9.5)$ & $211 / 15,125.9$ & $13.9(12.2-16.0)$ & $\mathrm{I}, 353 / 157,630.0$ & $8.6(8.1-9.1)$ \\
\hline Yes & $605 / 94,127.2$ & $6.4(5.9-7.0)$ & $\mid 23 / 17,520.3$ & $7.0(5.9-8.4)$ & $482 / 76,606.9$ & $6.3(5.8-6.9)$ \\
\hline \multicolumn{7}{|c|}{ Major mental illness ${ }^{a}$} \\
\hline No & $\mathrm{I}, 652 / 183,921.6$ & $9.0(8.6-9.4)$ & $229 / 20,043.6$ & II.4 (10.0-13.0) & I,423/I63,878.0 & $8.7(8.2-9.1)$ \\
\hline Yes & $517 / 82,961.4$ & $6.2(5.7-6.8)$ & $105 / 12,602.6$ & $8.3(6.9-10.1)$ & $4 \mid 2 / 70,358.8$ & $5.9(5.3-6.4)$ \\
\hline \multicolumn{7}{|c|}{ Mental health counseling ${ }^{a}$} \\
\hline No & $1,602 / 167,550.9$ & $10.0(9.6-10.5)$ & $213 / 17,516.5$ & $12.2(10.6-13.9)$ & I,389/I50,034.4 & $9.3(8.8-9.8)$ \\
\hline Yes & $567 / 99,332.1$ & $5.7(5.3-6.2)$ & $|2| /|5| 29.7$, & $8.0(6.7-9.6)$ & $446 / 84,202.4$ & $5.3(4.8-5.8)$ \\
\hline \multicolumn{7}{|c|}{ Mental health-counseling visits ${ }^{a}$} \\
\hline No & $1,602 / 167,550.9$ & $9.6(9.1-10.0)$ & $213 / 17,516.5$ & $12.2(10.6-13.9)$ & I,389/I50,034.4 & $9.3(8.8-9.8)$ \\
\hline I & I36/19,473.| & $7.0(5.9-8.3)$ & $27 / 2,987.4$ & $9.0(6.2-13.2)$ & $109 / 16,485.7$ & $6.6(5.5-8.0)$ \\
\hline$\geq 2$ & $431 / 79,859.1$ & $5.4(4.9-5.9)$ & $94 / 12,142.3$ & $7.7(6.3-9.5)$ & $337 / 67,716.8$ & $5.0(4.5-5.5)$ \\
\hline \multicolumn{7}{|l|}{ Depression $^{\mathrm{a}}$} \\
\hline No & I,358/I07,657.I & $12.6(12.0-13.3)$ & $195 / \mid I, 229.2$ & I7.4 (I5.|-20.0) & I,163/96,427.9 & $12.1(11.4-12.8)$ \\
\hline Yes & $811 / 159,225.9$ & $5.1(4.8-5.5)$ & $|39 / 2|, 4 \mid 7.0$ & $6.5(5.5-7.7)$ & $672 / 137,808.9$ & $4.9(4.5-5.3)$ \\
\hline \multicolumn{7}{|l|}{ Psychosis $^{a}$} \\
\hline No & I,989/239,710.5 & $8.3(7.9-8.7)$ & $291 / 28,040.2$ & $10.4(9.3-11.6)$ & I,698/2II,670.3 & $8.0(7.6-8.4)$ \\
\hline Yes & $180 / 27,172.6$ & $6.6(5.7-7.7)$ & $43 / 4,606.0$ & $9.3(6.9-12.6)$ & $137 / 22,566.6$ & $6.1(5.1-7.2)$ \\
\hline \multicolumn{7}{|c|}{ Problematic alcohol use $\mathrm{a}^{\mathrm{a}}$} \\
\hline No & I,569/189,785.2 & $8.3(7.9-8.7)$ & $240 / 22,458.1$ & $10.7(9.4-12.1)$ & I,392/167,327.I & $7.9(7.5-8.4)$ \\
\hline Yes & $600 / 77,097.8$ & $7.8(7.2-8.4)$ & $94 / 10,188.1$ & $9.2(7.5-11.3)$ & $506 / 66,909.7$ & $7.6(6.9-8.3)$ \\
\hline \multicolumn{7}{|l|}{ Active TB ${ }^{a}$} \\
\hline No & $2,161 / 265,687.8$ & $8.1(7.8-8.5)$ & $333 / 32,560.2$ & $10.2(9.2-11.4)$ & I,828/233,I27.6 & $7.8(7.5-8.2)$ \\
\hline Yes & $8 / 1,195.3$ & $6.7(3.3-13.4)$ & $\mathrm{I} / 86.0$ & II.6 (1.6-82.6) & $7 / 1,109.3$ & $6.3(3.0-13.2)$ \\
\hline \multicolumn{7}{|l|}{ Hepatitis $\mathbf{B}^{\mathbf{a}}$} \\
\hline No & I,963/247,85।.2 & $7.9(7.6-8.3)$ & $306 / 31,047.4$ & $9.9(8.8-11.0)$ & $\mathrm{I}, 657 / 2 \mathrm{I} 6,803.8$ & $7.6(7.3-8.0)$ \\
\hline Yes & $206 / 19,031.8$ & $10.8(9.4-12.4)$ & $28 / 1,598.7$ & $17.5(12.1-25.4)$ & $178 / 17,433 . \mid$ & $10.2(8.8-11.8)$ \\
\hline \multicolumn{7}{|l|}{ Sex } \\
\hline Female & $723 / 95,668.7$ & $7.6(7.0-8.1)$ & $|57 /| 5,284 . \mid$ & $10.3(8.8-12.0)$ & $566 / 80,384.6$ & $7.0(6.5-7.6)$ \\
\hline Male & I,446/I7I,2I4.4 & $8.4(8.0-8.9)$ & $|77 /| 7,362 . \mid$ & $10.2(8.8-11.8)$ & I,269/I53,852.2 & $8.2(7.8-8.7)$ \\
\hline Age at HCV d & & & & & & \\
\hline$<15$ years & $7 / 1,324.1$ & $5.3(2.5-I I . I)$ & $2 / 61.3$ & $32.6(8.2-130.4)$ & $5 / 1,262.8$ & $4.0(1.6-9.5)$ \\
\hline 15-24 years & $307 / 23,752$ & $12.9(\mid 1.6-14.5)$ & $105 / 8,300.6$ & $12.6(10.4-15.3)$ & $202 / 15,451.4$ & I3.I (II.4-15.0) \\
\hline 25-34 years & $802 / 76,680.7$ & $10.5(9.8-1 \mathrm{I} .2)$ & II5/I3,608.7 & $8.5(7.0-10.1)$ & $687 / 63,072.0$ & $10.9(10.1-11.7)$ \\
\hline $35-44$ years & $788 / 102,562.8$ & $7.7(7.2-8.2)$ & $88 / 7,925.6$ & II.I (9.0-13.7) & $700 / 94,637.2$ & $7.4(6.9-8.0)$ \\
\hline 45-54 years & $233 / 49,692.8$ & $4.7(4.1-5.3)$ & $21 / 2,337.0$ & $9.0(5.9-13.8)$ & $212 / 47,355.8$ & $4.5(3.9-5.1)$ \\
\hline$>54$ years & $32 / 12,870.7$ & $2.5(1.8-3.5)$ & $3 / 413.1$ & $7.3(2.3-22.5)$ & $29 / 12,457.7$ & $2.3(1.6-3.3)$ \\
\hline Year of HCV & & & & & & \\
\hline Before 2000 & I,578/I8I,223.5 & $8.7(8.3-9.1)$ & $|2| / \mid 0,640.8$ & II.4 (9.5-13.6) & I,457/I 70,582.6 & $8.5(8.1-9.0)$ \\
\hline 2000-2004 & $42 \mathrm{I} / 60,87 \mathrm{I} .4$ & $6.9(6.3-7.6)$ & I50/I3,998.| & $10.7(9.1-12.6)$ & $271 / 46,873.3$ & $5.8(5.1-6.5)$ \\
\hline $2005-2008$ & $122 / 19,485$ & $6.3(5.2-7.5)$ & $45 / 6,213.6$ & $7.2(5.4-9.7)$ & $77 / 13,27 \mid .3$ & $5.8(4.6-7.3)$ \\
\hline 2009-20II & $4 \mathrm{l} / 4,769.9$ & $8.6(6.3-11.7)$ & $17 / 1,606.7$ & $10.6(6.6-17.0)$ & $24 / 3,163.1$ & $7.6(5.1-11.3)$ \\
\hline After 20II & $7 / 533.4$ & I3.I (6.3-27.5) & $1 / 186.8$ & $5.4(0.8-38.0)$ & $6 / 346.5$ & $17.3(7.8-38.5)$ \\
\hline
\end{tabular}


Table 2 (Continued)

\begin{tabular}{|c|c|c|c|c|c|c|}
\hline \multirow[t]{2}{*}{ Variables } & \multicolumn{2}{|c|}{ HCV infection } & \multicolumn{2}{|c|}{$\mathrm{HCV}^{+}$seroconverters } & \multicolumn{2}{|c|}{$\mathrm{HCV}^{+}$-prevalent } \\
\hline & $\begin{array}{l}\text { n/person- } \\
\text { years }\end{array}$ & $\begin{array}{l}\text { Incidence } \\
(95 \% \text { CI) }\end{array}$ & $\begin{array}{l}\text { n/person- } \\
\text { years }\end{array}$ & $\begin{array}{l}\text { Incidence } \\
(95 \% \mathrm{Cl})\end{array}$ & $\begin{array}{l}\text { n/person- } \\
\text { years }\end{array}$ & $\begin{array}{l}\text { Incidence } \\
(95 \% \mathrm{Cl})\end{array}$ \\
\hline \multicolumn{7}{|l|}{ Urban } \\
\hline No & $|43 / 26,828|$. & $5.3(4.5-6.3)$ & $25 / 3,009.0$ & $8.3(5.6-12.3)$ & $118 / 23,819.2$ & $5.0(4.1-5.9)$ \\
\hline Yes & $2,007 / 236,434.3$ & $8.5(8.1-8.9)$ & $309 / 29,192.7$ & $10.6(9.5-11.8)$ & I,698/207,24I.5 & $8.2(7.8-8.6)$ \\
\hline Unknown & $19 / 3,260.6$ & $5.2(3.3-8.2)$ & 0 & 0 & $19 / 3,|76|$. & $6.0(3.8-9.4)$ \\
\hline
\end{tabular}

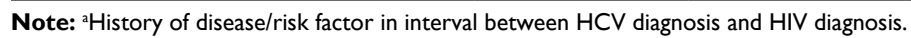

Abbreviations: BC, British Columbia; HCV, hepatitis C virus; IDU, injection-drug use; MSM, men who have sex with men; OST, opioid-substitution therapy; TB, tuberculosis.

A

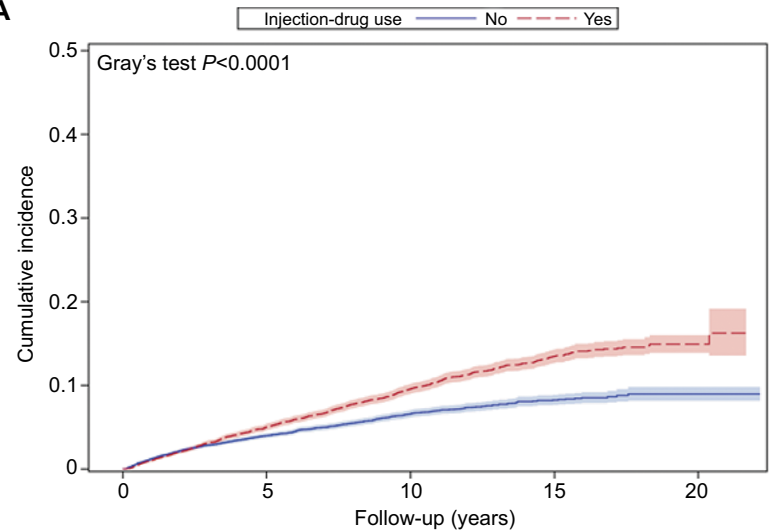

Number at risk

No $\quad 23,187$

Yes $\quad 12,890$

C

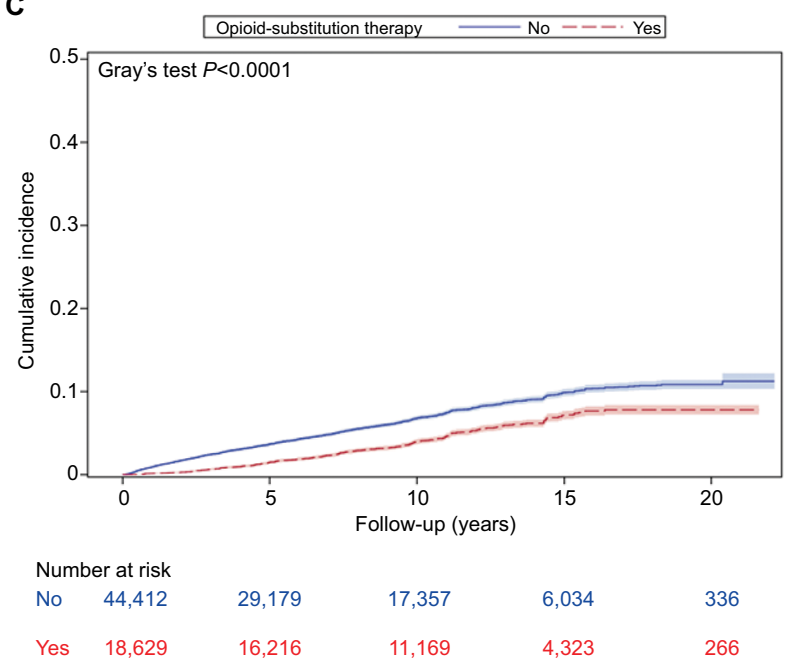

B

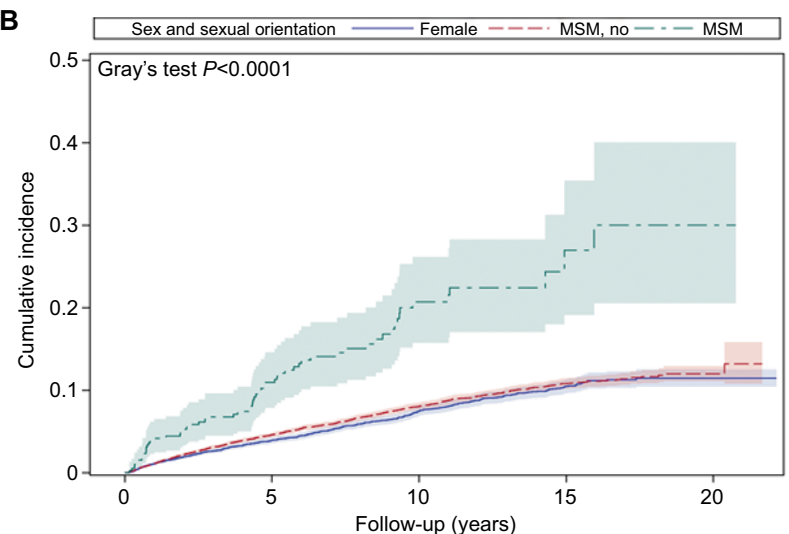

Number at risk

$\begin{array}{llllll}\text { Female } & 12,776 & 7,707 & 4,330 & 1,428 & 73\end{array}$

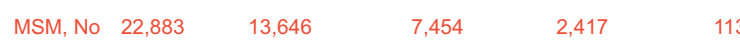

MSM, Yes 418

D

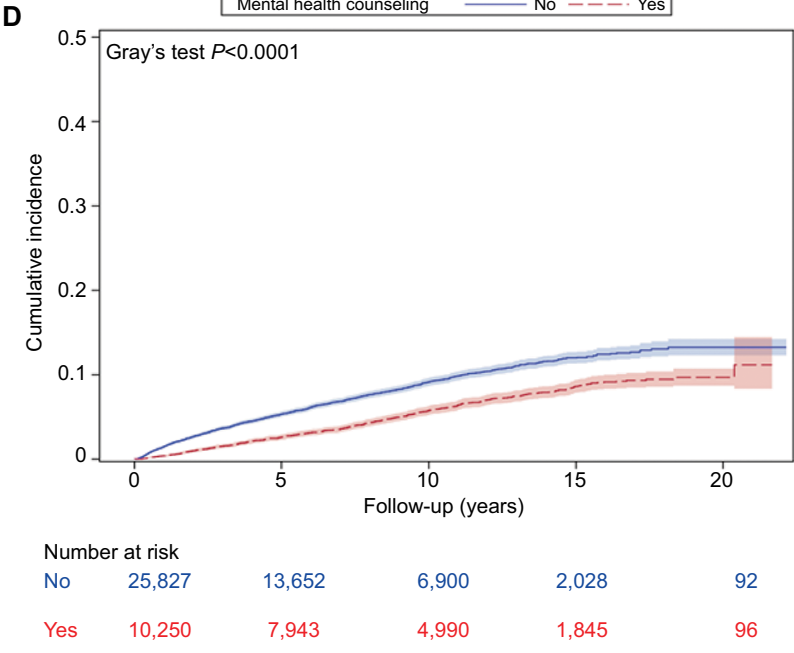

Figure 2 Cumulative incidence of HIV, British Columbia Hepatitis Testers Cohort, 1990-2013.

Note: (A) By injection-drug use; (B) by sex and sexual orientation; (C) by opioid-substitution therapy; and (D) by mental health counseling.

Abbreviation: MSM, men who have sex with men.

health counseling (adjusted HR 0.48, 95\% CI 0.43-0.53) were associated with reduced risk of HIV (Table 3). In a sensitivity analysis conducted using the number of mental health-counseling visits per year, an increase in the number of visits was associated with a linear increase in the reduction of
$\mathrm{HIV}$ risk for overall $\mathrm{HCV}^{+}$and $\mathrm{HCV}^{+}$-prevalent individuals, but not for $\mathrm{HCV}^{+}$seroconverters (Table S3).

In the model restricted to $\mathrm{HCV}^{+}$seroconverters, a similar pattern emerged as for all HCV diagnoses; however, the magnitude of the HRs was larger than in the model restricted 
Table 3 Multivariable Cox regression analysis of factors associated with time to HIV infection in the BC Hepatitis Testers Cohort

\begin{tabular}{|c|c|c|c|c|c|c|}
\hline & \multicolumn{2}{|l|}{ HCV infection } & \multicolumn{2}{|c|}{$\mathbf{H C V}^{+}$seroconverters } & \multicolumn{2}{|c|}{$\mathrm{HCV}^{+}$-prevalent } \\
\hline & Adjusted HR & $95 \% \mathrm{Cl}$ & Adjusted HR & $95 \% \mathrm{Cl}$ & Adjusted HR & $95 \% \mathrm{Cl}$ \\
\hline \multicolumn{7}{|c|}{ Age at HCV diagnosis } \\
\hline$<25$ years & 2.38 & $2.00-2.82$ & 1.32 & $0.83-2.08$ & 2.10 & I.72-2.55 \\
\hline $25-34$ years & 1.95 & $1.69-2.25$ & 0.81 & $0.51-1.27$ & 2.14 & $1.84-2.50$ \\
\hline $35-44$ years & 1.58 & $1.37-1.82$ & 1.23 & $0.78-1.95$ & 1.47 & $|.27-1.7|$ \\
\hline$\geq 45$ & 1 & & I & & I & \\
\hline \multicolumn{7}{|c|}{ Year of HCV diagnosis } \\
\hline Before 2000 & 1.37 & $0.65-2.91$ & 4.68 & $0.64-34.08$ & 0.97 & $0.43-2.19$ \\
\hline 2000-2004 & 0.78 & $0.37-1.67$ & 3.15 & $0.43-22.88$ & 0.60 & $0.26-1.36$ \\
\hline $2005-2008$ & 0.7 & $0.32-|.5|$ & 2.01 & $0.27-14.77$ & 0.49 & $0.21-1.14$ \\
\hline 2009-20II & 0.81 & $0.36-1.82$ & 2.31 & $0.30-17.54$ & 0.57 & $0.23-1.40$ \\
\hline After $201 \mathrm{I}$ & 1 & & I & & I & \\
\hline \multicolumn{7}{|l|}{ Urban } \\
\hline No & I & & I & & I & \\
\hline Yes & 1.45 & $1.22-1.72$ & 1.19 & $0.79-1.80$ & $\mathrm{I} .47$ & $1.22-1.78$ \\
\hline Unknown & 0.75 & $0.45-1.24$ & 0 & 0 & 0.86 & $0.51-1.44$ \\
\hline \multicolumn{7}{|c|}{ Sex and sexual orientation } \\
\hline Male, not MSM & 1 & & 1 & & 1 & \\
\hline Male, yes MSM & 2.78 & $2.14-3.61$ & 5.62 & $3.12-10.14$ & 2.60 & $1.93-3.48$ \\
\hline Female & 0.81 & $0.73-0.89$ & 0.96 & $0.76-1.20$ & 0.79 & $0.7 I-0.88$ \\
\hline HIV tests per year & 1.57 & $1.54-1.59$ & 1.46 & $|.4|-|.5|$ & 2.01 & $1.96-2.06$ \\
\hline \multicolumn{7}{|l|}{ IDU } \\
\hline No & I & & I & & I & \\
\hline Yes & 1.47 & $1.33-1.63$ & 1.13 & $0.87-1.47$ & 1.61 & $1.45-1.79$ \\
\hline OST $^{\mathbf{a}}$ & 0.59 & $0.52-0.67$ & 0.67 & $0.50-0.91$ & 0.56 & $0.48-0.65$ \\
\hline \multicolumn{7}{|c|}{ Problematic alcohol use $^{a}$} \\
\hline No & 1 & & I & & I & \\
\hline Yes & 0.99 & $0.89-1.10$ & 0.99 & $0.77-1.29$ & 0.84 & $0.75-0.94$ \\
\hline \multicolumn{7}{|c|}{ Mental health counseling ${ }^{a}$} \\
\hline No & I & & I & & I & \\
\hline Yes & 0.48 & $0.43-0.53$ & 0.56 & $0.44-0.72$ & 0.44 & $0.39-0.50$ \\
\hline \multicolumn{7}{|l|}{ Active TB ${ }^{a}$} \\
\hline No & 1 & & I & & I & \\
\hline Yes & 0.8 & $0.40-1.61$ & $\mathrm{I} .47$ & $0.20-10.60$ & 0.77 & $0.37-1.63$ \\
\hline \multicolumn{7}{|l|}{ Hepatitis B ${ }^{a}$} \\
\hline No & I & & I & & I & \\
\hline Yes & 1.34 & $1.16-1.55$ & 1.85 & $1.24-2.75$ & 1.27 & $1.08-1.48$ \\
\hline \multicolumn{7}{|c|}{ Material deprivation quintile at time of test } \\
\hline QI (most privileged) & I & & I & & I & \\
\hline Q2 & 0.69 & $0.58-0.82$ & 0.67 & $0.44-1.02$ & 0.92 & $0.76-1.11$ \\
\hline Q3 & 0.73 & $0.6 \mathrm{I}-0.86$ & 0.68 & $0.46-1.02$ & 0.81 & $0.67-0.98$ \\
\hline Q4 & 0.85 & $0.73-0.99$ & 0.68 & $0.47-0.99$ & 0.96 & $0.81-1.13$ \\
\hline Q5 (most deprived) & 1.09 & $0.95-1.25$ & 0.72 & $0.51-1.02$ & 1.23 & $1.06-1.43$ \\
\hline Unknown & 1.54 & $1.13-2.11$ & 0.24 & $0.54-0.19$ & 1.88 & $1.35-2.62$ \\
\hline
\end{tabular}

Note: ${ }^{a}$ History of disease/risk factor in interval between HCV diagnosis and HIV diagnosis.

Abbreviations: BC, British Columbia; HCV, hepatitis C virus; IDU, injection-drug use; MSM, men who have sex with men; OST, opioid-substitution therapy; TB, tuberculosis.

to $\mathrm{HCV}^{+}$-prevalent individuals for MSM status and $\mathrm{HBV}$ infection (Table 3). No association was found between urban residence and $\mathrm{HIV}$ infection among $\mathrm{HCV}^{+}$seroconverters. In analysis on $\mathrm{HCV}^{+}$-prevalent individuals, age at $\mathrm{HCV}$ diagnosis, urban residence, MSM, and IDU were significantly associated with risk of HIV infection. However, the strength of association for IDU and material deprivation was higher among $\mathrm{HCV}^{+}$-prevalent individuals than that for $\mathrm{HCV}^{+}$seroconverters. In addition, the effect of OST and mental health counseling on risk of HIV infection was more pronounced among $\mathrm{HCV}^{+}$-prevalent individuals compared to $\mathrm{HCV}^{+}$ seroconverters (Table 3). The interaction of OST and mental health counseling was not significant $(P=0.075)$. In addition, we evaluated the effectiveness of mental health counseling 
among $\mathrm{HCV}^{+}$people with mental illness and without mental illness separately. Among people who had major mental illness, there was a $61 \%$ reduction in the risk of HIV compared to a $44 \%$ reduction in HIV risk among those who did not have history of major mental illness (Table S4).

\section{Discussion}

In this large population-based cohort study in a setting of widespread OST availability, the incidence of HIV infection was higher among $\mathrm{HCV}^{+}$seroconverters than $\mathrm{HCV}^{+}$-prevalent individuals. The risk of HIV infection was higher among MSM, PWID, urban residents, and those with HBV coinfection. OST and engagement in mental health counseling significantly reduced the risk of acquiring HIV infection. These interventions have important implications in prevention of HIV transmission in various settings globally.

Although earlier studies have identified a lower risk of HIV among PWID on OST, ${ }^{11,12}$ to our knowledge, this is the first study examining the association of OST and mental health counseling on the risk of HIV among $\mathrm{HCV}^{+}$individuals in a large population-based cohort. We showed that OST was associated with a $41 \%$ reduction and mental health counseling with a $52 \%$ reduction in $\mathrm{HIV}$ risk among $\mathrm{HCV}^{+}$individuals. A systematic review and meta-analysis of the effect of OST and HIV transmission reported a 54\% reduction in HIV risk among PWID, ${ }^{12}$ whereas another study from BC on methadone maintenance therapy and HIV-infection risk reported a reduction of $36 \%$ among PWID. ${ }^{11}$ However, both of these studies included PWID who did not have HCV infection or had not been tested for HCV. These interventions, coupled with other harm-reduction activities like syringe distribution and the expansion of safe injection sites, could have a major impact on $\mathrm{HIV}$-infection risk among $\mathrm{HCV}^{+}$populations, particularly PWID.

Currently, OST is available as a low-threshold treatment across most regions of $\mathrm{BC}$ for the management of opioid addiction. However, the availability of OST is limited in many settings, including developed and developing countries across the world. Globally, an estimated $6 \%-12 \%$ of PWID receive OST, ${ }^{20}$ though coverage is even lower among countries with emerging epidemics of HIV among PWID. A survey of 21 of these countries from different regions of the world on HIVprevention and treatment-service availability reported OST coverage of $<3 \%$ among PWID. ${ }^{21}$ In addition, mental healthservices provision for the general population, especially in low- and middle-income countries is limited, ${ }^{22}$ and is even lower for people with substance-use disorders, ${ }^{23}$ particularly PWID. Data from the World Mental Health Survey estimate that only $1 \%$ of people with substance-use disorders in lowand middle-income countries have treatment coverage for these disorders. ${ }^{23}$ Treatment of mental illnesses among PWID has been identified as an important part of HIV prevention. ${ }^{24}$ In this context, mental health counseling plays an important role in HIV prevention. Psychotherapy, particularly behavioral interventions, similar to the effect of OST, has been shown to reduce HIV-acquisition risk in a number of studies on $\mathrm{MSM}^{25}$ and PWID. ${ }^{26,27}$ However, varying evidence exists on the efficacy of single vs multiple psychosocial interventions and educational interventions. Some meta-analyses support single-session behavioral interventions ${ }^{28}$ for preventing STIs, including HIV, whereas another meta-analysis reported reduction in HIV-risk behavior in PWID with multiple sessions and educational interventions. ${ }^{26}$ Our study provides evidence with respect to continued psychosocial support for reduction in HIV risk in a high-risk population of HCV-infected individuals. However, the linear relationship between reduction in HIV risk and the number of mental health-counseling visits was not apparent for $\mathrm{HCV}^{+}$seroconverters (Table S3). As $\mathrm{HCV}^{+}$seroconverters exhibit higher-risk behavior like active IDU, the effect of repeated mental health-counseling visits on reducing HIV risk may have been diluted. Additionally, individuals with more severe mental disorders like schizophrenia and bipolar disorder are less likely to attend followup visits for mental health care, due to mental illness-related stigma. ${ }^{29,30} \mathrm{HCV}^{+}$seroconverters in our study had a higher proportion of major mental illness, which includes disorders like schizophrenia and bipolar disorder, and may have been less likely to continue with mental health counseling. However, all studies recommend a combination of HIV-prevention strategies to maximize the effect of psychosocial interventions on HIV-risk reduction..$^{25-28}$ The availability and use of OST, along with mental health counseling and coupled with other harm-reduction interventions, could reduce the risk of such blood-borne infections as HIV, as shown in this study. Similar interventions have shown the potential to reduce the risk of $\mathrm{HCV}$ in previous research. ${ }^{14,31,32}$

In our cohort, $6 \%$ of HCV-infected individuals developed HIV infection, with an incidence rate of 8.1/1,000 person years. However, there are no data on HIV incidence among $\mathrm{HCV}$-infected populations. Studies reporting HIV incidence present estimates of 1.66-6.23/100 person-years among PWID $^{33}$ and as high as 35.2/100 person-years among MSM. ${ }^{34}$ This wider range of estimates could be due to differences in study populations, follow-up time, testing patterns, risk activities, and availability and impact of harm-reduction programs and interventions. 
$\mathrm{HCV}^{+}$seroconverters had a higher incidence of HIV infection compared to $\mathrm{HCV}^{+}$-prevalent individuals. This relates to differences in the magnitude of risk factors and population composition, which varied across these two groups. $\mathrm{HCV}^{+}$seroconverters were younger, more socially and materially deprived, and had higher illicit-drug use, IDU, and major mental illnesses than $\mathrm{HCV}^{+}$-prevalent individuals. Seroconverters have higher-risk behavior, which is why they are followed for testing and detected as seroconverters, while prevalent infections represent a mix of high- and low-risk individuals. This may explain the difference between seroconverters and prevalent individuals in terms of magnitude of their risk activities.

In our study, being MSM was associated with a higher risk of HIV infection. ${ }^{35}$ IDU and high-risk sexual behavior among MSM have been documented as major predictors for acquisition of HIV. "Chemsex" or sexualized drug use among MSM has been associated with higher risk-taking behaviors and STIs, including HIV and HCV infection. ${ }^{37}$ However, MSM among $\mathrm{HCV}^{+}$seroconverters were at higher risk of HIV than MSM among $\mathrm{HCV}^{+}$-prevalent individuals. As stated, in our cohort, $\mathrm{HCV}^{+}$seroconverters were younger and had a higher proportion of IDU, problematic alcohol use, and mental illness. Previous studies have reported a high incidence of HIV among young MSM, ${ }^{38}$ especially related to drug use ${ }^{39}$ and unprotected sex. ${ }^{40}$ Services addressing substance use and mental illness in a stigma-free environment may reduce the risk of subsequent HIV infection among MSM. ${ }^{41,42}$

In our cohort, HIV indicator conditions, namely TB and $\mathrm{HBV}$, were significantly associated with a higher risk of $\mathrm{HIV}^{43}$ Among these diseases, HBV has historically been a marker for behavior associated with potentially increased risk of HIV transmission. This represents an opportunity for early detection and treatment of HIV to reduce HIV-related morbidity and transmission. ${ }^{44}$

Our outcome variable, time to HIV infection, also included individuals who did not have a last negative HIV test prior to a first HIV-positive test; however, a sensitivity analysis conducted after removal of these individuals yielded similar results as our main model (Table S5). Mental health counseling in $\mathrm{BC}$ is covered through a Medical Services Plan which includes all services that are billed by health care providers. However, if a service was provided without a fee from a service provider, then this information would not be captured and may lead to an underestimation of receipt of counseling services. Additionally, mental health counseling does not differentiate between the severity and type of mental illnesses/conditions and may not have the same effect in all high-risk populations. The major mental illness variable is based on a psychiatric visit, may include more severe mental illness diagnosis, and may underestimate mental illness and lead to misclassification of mental illness. Also, as these indicators are based on administrative databases, there is a possibility of misclassification. However, as expected, results showed stronger impact among those with mental illness compared to those without documented major mental illness (Table S4). The assessment of such risk factors as IDU and problematic alcohol use was based on diagnostic codes in administrative health-services databases. Our definitions were based on validations done previously; ${ }^{45}$ however, there could be some misclassification and underestimation of effect. Furthermore, some of the interactions could not be evaluated, because of smaller samples, eg, MSM and IDU. All prescriptions that are dispensed in $\mathrm{BC}$ are recorded in a centralized system including OST, thereby capturing all dispensed OST. As causal inferences based on intervention effects in observational studies are prone to biases, future research using experimental designs is needed to validate our findings.

\section{Conclusion}

In this large population-based cohort of $\mathrm{HCV}$-infected individuals, $\mathrm{HCV}^{+}$seroconverters, PWID, MSM, and those coinfected with HBV had a higher risk of HIV infection, while OST and mental health counseling were associated with reduced risk of HIV. These data provide further support for the role of OST and mental health services in reducing transmission of bloodborne infections. Improving access to OST and mental health services could prevent transmission of HIV and other bloodborne infections, especially in settings where access is limited.

\section{Author contributions}

ZAB, NZJ, JAB, GO, RFB, MWT, MK, DG, MG, JW, and MM conceived the study. ZAB and NZJ designed the study. $\mathrm{AY}, \mathrm{NS}$, and $\mathrm{SW}$ assisted in data management. ZAB, NS, SW, and NZJ formulated the statistical analysis of the study. ZAB prepared the original draft of the paper. ZAB, NS, SW, MK, DG, MG, JW, AY, MA, HS, JAB, DR, TC, MM, MWH, GO, RFB, MWT, MK, and NZJ revised the paper critically for important intellectual content. All authors contributed toward data analysis, drafting and revising the paper and agree to be accountable for all aspects of the work.

\section{Acknowledgments}

We acknowledge the assistance of BCCDC, PHSA Performance Measurement and Reporting, Information Analysts, 
Ministry of Health Data Access, Research, and Stewardship, Medical Services Plan (MSP), Discharge Abstract Database (DAD) and Medical Beneficiary and Pharmaceutical Services program areas, BC Ministry of Health, and BC Cancer Agency and their staff involved in data access, procurement, and management. All inferences, opinions, and conclusions drawn in this publication are those of the author(s), and do not necessarily reflect the opinions or policies of the $\mathrm{BC}$ Ministry of Health. This work was supported by the BC Center for Disease Control, agencies contributing data to the study, and the Canadian Institutes of Health Research (grants PHE-141773 and NHC-142832). The funder had no role in study design, data collection or analysis, decision to publish, or preparation of the manuscript.

\section{Disclosure}

MK has received grant funding via his institution from Roche Molecular Systems, Boehringer Ingelheim, Merck, Siemens Healthcare Diagnostics, and Hologic. The authors report no other conflicts of interest in this work.

\section{References}

1. World Health Organization. HIV and hepatitis coinfections. 2017. Available from: http://www.who.int/hiv/topics/hepatitis/hepatitisinfo/ en. Accessed January 27, 2017.

2. Fernández-Montero JV, Barreiro P, de Mendoza C, Labarga P, Soriano V. Hepatitis $\mathrm{C}$ virus coinfection independently increases the risk of cardiovascular disease in HIV-positive patients. JViral Hepat. 2016;23(1):47-52.

3. Mocroft A, Neuhaus J, Peters L, et al. Hepatitis B and C co-infection are independent predictors of progressive kidney disease in HIV-positive, antiretroviral-treated adults. PLoS One. 2012;7(7):e40245.

4. Butt ZA, Wilkins MJ, Hamilton E, Todem D, Gardiner JC, Saeed M. Survival of HIV-positive individuals with hepatitis B and C infection in Michigan. Epidemiol Infect. 2014;142(10):2131-2139.

5. Klein MB, Rollet-Kurhajec KC, Moodie EE, et al. Mortality in HIVhepatitis C co-infected patients in Canada compared to the general Canadian population (2003-2013). AIDS. 2014;28(13):1957-1965.

6. Simon D, Michita RT, Béria JU, Tietzmann DC, Stein AT, Lunge VR. Alcohol misuse and illicit drug use are associated with HCV/HIV coinfection. Epidemiol Infect. 2014;142(12):2616-2623.

7. Bonacini M. Alcohol use among patients with HIV infection. Ann Hepatol. 2011;10(4):502-507.

8. Rauch A, Rickenbach M, Weber R, et al. Unsafe sex and increased incidence of hepatitis $\mathrm{C}$ virus infection among HIV-infected men who have sex with men: the Swiss HIV Cohort Study. Clin Infect Dis. 2005;41(3):395-402.

9. van de Laar TJ, van der Bij AK, Prins M, et al. Increase in HCV incidence among men who have sex with men in Amsterdam most likely caused by sexual transmission. J Infect Dis. 2007;196(2):230-238.

10. Rourke SB, Sobota M, Tucker R, et al. Social determinants of health associated with hepatitis $\mathrm{C}$ co-infection among people living with HIV: results from the Positive Spaces, Healthy Places study. Open Med. 2011;5(3):e120-e131.

11. Ahamad K, Hayashi K, Nguyen P, et al. Effect of low-threshold methadone maintenance therapy for people who inject drugs on HIV incidence in Vancouver, BC, Canada: an observational cohort study. Lancet HIV. 2015;2(10):e445-e450.
12. Macarthur GJ, Minozzi S, Martin N, et al. Opiate substitution treatment and HIV transmission in people who inject drugs: systematic review and meta-analysis. BMJ. 2012;345:e5945.

13. Kerr T, Small W, Buchner C, et al. Syringe sharing and HIV incidence among injection drug users and increased access to sterile syringes. $\mathrm{Am}$ J Public Health. 2010;100(8):1449-1453.

14. Islam N, Krajden M, Shoveller J, et al. Incidence, risk factors, and prevention of hepatitis $\mathrm{C}$ reinfection: a population-based cohort study. Lancet Gastroenterol Hepatol. 2017;2(3):200-210.

15. Janjua NZ, Kuo M, Chong M, et al. Assessing hepatitis $C$ burden and treatment effectiveness through the British Columbia Hepatitis Testers Cohort (BC-HTC): design and characteristics of linked and unlinked participants. PLoS One. 2016;11(3): 0150176.

16. Janjua NZ, Yu A, Kuo M, et al. Twin epidemics of new and prevalent hepatitis $\mathrm{C}$ infections in Canada: $\mathrm{BC}$ Hepatitis Testers Cohort. BMC Infect Dis. 2016;16:334.

17. Nosyk B, Colley G, Yip B, et al. Application and validation of casefinding algorithms for identifying individuals with human immunodeficiency virus from administrative data in British Columbia, Canada. PLoS One. 2013;8(1):e54416.

18. Pampalon R, Hamel D, Gamache P, Philibert MD, Raymond G, Simpson A. An area-based material and social deprivation index for public health in Québec and Canada. Can J Public Health. 2012;103(8 Supp1 2):S17-S22.

19. Nosyk B, Macnab YC, Sun H, et al. Proportional hazards frailty models for recurrent methadone maintenance treatment. Am J Epidemiol. 2009;170(6):783-792.

20. Mathers BM, Degenhardt L, Ali H, et al. HIV prevention, treatment, and care services for people who inject drugs: a systematic review of global, regional, and national coverage. Lancet. 2010;375(9719):1014-1028.

21. Petersen Z, Myers B, van Hout MC, Plüddemann A, Parry C. Availability of HIV prevention and treatment services for people who inject drugs: findings from 21 countries. Harm Reduct J. 2013;10:13.

22. Rathod S, Pinninti N, Irfan M, et al. Mental health service provision in low- and middle-income countries. Health Serv Insights. 2017; 10:117863291769435.

23. Degenhardt L, Glantz M, Evans-Lacko S, et al. Estimating treatment coverage for people with substance use disorders: an analysis of data from the World Mental Health Surveys. World Psychiatry. 2017;16(3): 299-307.

24. Buckingham E, Schrage E, Cournos F. Why the treatment of mental disorders is an important component of HIV prevention among people who inject drugs. Adv Prev Med. 2013;2013:690386.

25. Koblin B, Chesney M, Coates T. Effects of a behavioural intervention to reduce acquisition of HIV infection among men who have sex with men: the EXPLORE randomised controlled study. Lancet. 2004;364(9428):41-50.

26. Meader N, Semaan S, Halton M, et al. An international systematic review and meta-analysis of multisession psychosocial interventions compared with educational or minimal interventions on the HIV sex risk behaviors of people who use drugs. AIDS Behav. 2013;17(6):1963-1978.

27. Gilchrist G, Swan D, Widyaratna K, et al. A systematic review and meta-analysis of psychosocial interventions to reduce drug and sexual blood borne virus risk behaviours among people who inject drugs. AIDS Behav. 2017;21(7):1791-1811.

28. Eaton LA, Huedo-Medina TB, Kalichman SC, et al. Meta-analysis of single-session behavioral interventions to prevent sexually transmitted infections: implications for bundling prevention packages. Am J Public Health. 2012;102(11):e34-e44.

29. Lawrence D, Kisely S. Inequalities in healthcare provision for people with severe mental illness. J Psychopharmacol. 2010;24(4 Suppl):61-68.

30. Knaak S, Szeto A, Fitch K, Modgill G, Patten S. Stigma towards borderline personality disorder: effectiveness and generalizability of an anti-stigma program for healthcare providers using a pre-post randomized design. Borderline Personal Disord Emot Dysregul. 2015;2:9. 
31. Hagan H, Pouget ER, des Jarlais DC. A systematic review and metaanalysis of interventions to prevent hepatitis $C$ virus infection in people who inject drugs. J Infect Dis. 2011;204(1):74-83.

32. Turner KM, Hutchinson S, Vickerman P, et al. The impact of needle and syringe provision and opiate substitution therapy on the incidence of hepatitis $\mathrm{C}$ virus in injecting drug users: pooling of $\mathrm{UK}$ evidence. Addiction. 2011;106(11):1978-1988.

33. Aspinall EJ, Nambiar D, Goldberg DJ, et al. Are needle and syringe programmes associated with a reduction in HIV transmission among people who inject drugs: a systematic review and meta-analysis. Int $J$ Epidemiol. 2014;43(1):235-248.

34. Sanders EJ, Okuku HS, Smith AD, et al. High HIV-1 incidence, correlates of HIV-1 acquisition, and high viral loads following seroconversion among MSM. AIDS. 2013;27(3):437-446.

35. Bini EJ, Currie SL, Shen H, et al. National multicenter study of HIV testing and HIV seropositivity in patients with chronic hepatitis $\mathrm{C}$ virus infection. J Clin Gastroenterol. 2006;40(8):732-739.

36. Beyrer C, Baral SD, van Griensven F, et al. Global epidemiology of HIV infection in men who have sex with men. Lancet. 2012;380(9839):367-377.

37. Hegazi A, Lee MJ, Whittaker W, et al. Chemsex and the city: sexualised substance use in gay bisexual and other men who have sex with men attending sexual health clinics. Int J STD AIDS. 2017;28(4):362-366.

38. Balaji AB, Bowles KE, Le BC, Paz-Bailey G, Oster AM. High HIV incidence and prevalence and associated factors among young MSM, 2008. AIDS. 2013;27(2):269-278.
39. Freeman P, Walker BC, Harris DR, et al. Methamphetamine use and risk for HIV among young men who have sex with men in 8 US cities. Arch Pediatr Adolesc Med. 2011;165(8):736-740.

40. Mustanski BS, Newcomb ME, du Bois SN, Garcia SC, Grov C. HIV in young men who have sex with men: a review of epidemiology, risk and protective factors, and interventions. J Sex Res. 2011;48(2-3): 218-253.

41. Johnson WD, Diaz RM, Flanders WD, et al. Behavioral interventions to reduce risk for sexual transmission of HIV among men who have sex with men. Cochrane Database Syst Rev. 2008;3:CD001230.

42. Herbst JH, Beeker C, Mathew A, et al. The effectiveness of individual-, group-, and community-level HIV behavioral risk-reduction interventions for adult men who have sex with men: a systematic review. Am J Prev Med. 2007;32(4 Suppl):38-67.

43. Søgaard OS, Lohse N, Østergaard L, et al. Morbidity and risk of subsequent diagnosis of HIV: a population based case control study identifying indicator diseases for HIV infection. PLoS One. 2012;7(3):e32538

44. Joore IK, Arts DL, Kruijer MJ, et al. HIV indicator condition-guided testing to reduce the number of undiagnosed patients and prevent late presentation in a high-prevalence area: a case-control study in primary care. Sex Transm Infect. 2015;91(7):467-472.

45. Janjua NZ, Islam N, Kuo M, et al. Identifying injection drug use and estimating population size of people who inject drugs using healthcare administrative datasets. Int J Drug Policy. 2018;55:31-39. 


\section{Supplementary materials}

Table SI Criteria and data sources for the BC Hepatitis Testers Cohort (BC-HTC)

\section{Criteria for inclusion in BC-HTC}

All individuals:

- tested at centralized provincial laboratory for HCV or HIV, or

- reported by BC public health as a confirmed case of HCV, or

- reported in BC enhanced-surveillance system as a confirmed case of HIV or AIDS (all reports), or

- reported by BC public health as a confirmed case of HBV, or

- included in BC Enhanced Strain Surveillance System (EHSSS) as an acute HBV or HCV case.

All individuals meeting at least one of these criteria were linked internally across all their tests and case reports. Those with a valid personal health number (PHN) were then sent for deterministic linkage with province-wide cancer and Ministry of Health (MoH) data sets.

\begin{tabular}{|c|c|}
\hline Provincial communicable-disease data sources & Data date ranges \\
\hline BCCDC-PHL HIV laboratory-testing data sets (tests: ELISA, Western blot, NAAT, p24, culture) & $1988-2013$ \\
\hline BCCDC-PHL HCV laboratory-testing data sets (tests: antibody, HCV RNA, genotyping) & $1992-2013$ \\
\hline HIVIAIDS Information System (HAISYS) (public health HIVIAIDS case reports) & $1980-2013$ \\
\hline Integrated Public Health information System (iPHIS; public health case reports of HCV, HBV, and TB) & $1990-2013$ \\
\hline Enhanced Strain Surveillance System (EHSSS; risk-factor data on a subset of acute HCV and acute HBV cases) & $2000-2013$ \\
\hline Cancer and $\mathrm{MoH}$ administrative data sources & Data date ranges \\
\hline BC Cancer Registry (BCCR; primary tumor registry, excludes metastatic cancers) & $1970-2012$ \\
\hline Discharge Abstracts Dataset (DAD; hospitalization records)' & $|985-2013 Q|$ \\
\hline Medical Services Plan (MSP; physician diagnostic and billing data) ${ }^{2}$ & $1990-2012$ \\
\hline PharmaCare/PharmaNet (Pharma; prescription-drug dispensations) ${ }^{3,4}$ & $1985-20 \mid 2$ \\
\hline BC Vital Statistics (VS; deaths registry) ${ }^{5}$ & $1985-2013$ \\
\hline
\end{tabular}

Abbreviations: BC, British Columbia; BCCDC-PHL, BC Centre for Disease Control Public Health Laboratory; ELISA, enzyme-linked immunosorbent assay; HCV, hepatitis $C$ virus; NAAT, nucleic acid amplification test; TB, tuberculosis. 
Table S2 Definitions for comorbid conditions in the BC Hepatitis Testers Cohort (BC-HTC)

I. Major mental illness diagnosis

Major mental illness flagged at first occurrence of either one hospitalization diagnostic code or two MSP diagnostic codes from a psychiatrist visit for schizophrenic, bipolar, delusional, inorganic psychotic, adjustment, anxiety, dissociative, personality, and major depressive disorders

Physician billing data: MSP ICD9 diagnostic codes starting with 295-298, 300-30I, 308-309, 3II and claim specialty 3

Hospitalization data: DADI/ICD9-CM: starting with 295-298, 300-30I, 308-309, 3I I; DAD2/ICDI0-CA: starting with F20-F25, F28-F34, F38-F45,

F48, F60-F6I

\section{Problematic alcohol use}

Problematic alcohol use defined at first occurrence of one MSP or one hospitalization code for major alcohol-related diagnoses, including alcoholic mental disorders and dependence/abuse syndromes, alcoholic polyneuropathy, myopathy, cardiomyopathy, pseudo-Cushing's syndrome, or discharge to alcohol rehabilitation, counseling, or surveillance

Physician billing data: MSP ICD9 diagnostic codes starting with 29I, 303, 3050, 3575, 4255

Hospitalization data: DADI/ICD9-CM starting with 29I, 303, 3050, 3575, 4255; DAD2/ICDI0-CA starting with FI0, E244, G3I2, G62I, G72I, I426, Z502, Z714

\section{Illicit-drug use}

Illicit-drug use defined at first occurrence of one MSP or one hospitalization diagnostic code for major drug-related diagnoses involving addiction, dependence, and drug-induced mental disorders, illicit drug use, or illicit use of prescribed drugs, including hallucinogens, barbiturates/tranquillizers, sedatives, hypnotics, anxiolytics, opioids, cocaine, amphetamine, and volatile solvents, or discharge to drug rehabilitation, counseling, surveillance, or methadone/buprenorphine substitution treatment

Physician billing data: MSP ICD9 diagnostic codes starting with 292, 304, 3053-3057 or exact codes V6542 or 39

Hospitalization data: DADI/ICD9-CM starting with 292, 304, 3053-3057 or exact code V6542; DAD2/ICDI0 CA starting with FII-FI6, FI8-FI9,

Z7I5, Z503

\section{Mental health counseling}

Mental health counseling and rehabilitation defined at first occurrence of one MSP fee-item code for consultations and psychotherapy sessions related to mental health problems within study period

MSP fee-item code: 600-699, 60607-60645

\section{Opioid-substitution therapy}

Opioid-substitution therapy defined at first occurrence of two MSP codes or one PharmaNet DIN/PIN code for methadone/buprenorphine substitution treatment

Physician billing data: MSP ICD9 fee item 39

PharmaNet DIN/PIN: 2242963, 2242964, 999792, 66999990-3, 66999997, 66999998, 66999999, 67000000, 2295695, 2295709

Abbreviations: BC, British Columbia; MSP, Medical Services Plan; DIN, drug-identification number; PIN, product-identification number; DAD, Discharge Abstract Database; ICD, International Classification of Diseases. 
Table S3 Multivariable Cox regression analysis of factors associated with time to HIV infection in the BC Hepatitis Testers Cohort

\begin{tabular}{|c|c|c|c|c|c|c|}
\hline \multirow[t]{2}{*}{ Variables } & \multicolumn{2}{|l|}{ HCV infection } & \multicolumn{2}{|c|}{$\mathrm{HCV}^{+}$seroconverters } & \multicolumn{2}{|c|}{$\mathrm{HCV}^{+}$-prevalent } \\
\hline & Adjusted HR & $95 \% \mathrm{Cl}$ & Adjusted HR & $95 \% \mathrm{Cl}$ & Adjusted HR & $95 \% \mathrm{Cl}$ \\
\hline \multicolumn{7}{|c|}{ Age at HCV diagnosis } \\
\hline$<25$ years & 2.36 & $1.99-2.80$ & 1.32 & $0.83-2.09$ & 2.07 & $1.70-2.52$ \\
\hline $25-34$ years & 1.95 & $1.69-2.25$ & 0.79 & $0.5 \mathrm{I}-1.26$ & 2.13 & $1.83-2.48$ \\
\hline $35-44$ years & 1.58 & $1.37-1.82$ & 1.23 & $0.78-1.95$ & 1.47 & $1.26-1.7 \mid$ \\
\hline$\geq 45$ years & I & & 1 & & I & \\
\hline \multicolumn{7}{|c|}{ Year of HCV diagnosis } \\
\hline Before 2000 & 1.37 & $0.65-2.90$ & 4.71 & $0.64-34.27$ & 0.97 & $0.43-2.19$ \\
\hline 2000-2004 & 0.78 & $0.36-1.66$ & 3.16 & $0.43-22.95$ & 0.60 & $0.26-1.36$ \\
\hline $2005-2008$ & 0.70 & $0.32-|.5|$ & 2.02 & $0.27-14.84$ & 0.49 & $0.21-1.14$ \\
\hline 2009-20II & 0.81 & $0.36-1.82$ & 2.32 & $0.30-17.58$ & 0.57 & $0.23-1.40$ \\
\hline After 20I I & I & & I & & 1 & \\
\hline \multicolumn{7}{|l|}{ Urban } \\
\hline No & I & & I & & I & \\
\hline Yes & 1.45 & $1.22-1.72$ & 1.19 & $0.79-1.80$ & 1.48 & $1.23-1.79$ \\
\hline Unknown & 0.75 & $0.45-1.24$ & 0 & 0 & 0.87 & $0.52-1.46$ \\
\hline \multicolumn{7}{|c|}{ Sex and sexual orientation } \\
\hline Male, not MSM & I & & I & & 1 & \\
\hline Male, yes MSM & 2.78 & $2.14-3.61$ & 5.63 & $3.12-10.15$ & 2.61 & $1.94-3.50$ \\
\hline Female & 0.81 & $0.74-0.89$ & 0.96 & $0.76-1.20$ & 0.79 & $0.71-0.88$ \\
\hline HIV tests per year & 1.56 & $1.54-1.59$ & 1.46 & $|.4|-|.5|$ & 2.02 & $1.97-2.06$ \\
\hline \multicolumn{7}{|l|}{ IDU } \\
\hline No & I & & I & & I & \\
\hline Yes & 1.48 & $1.34-1.63$ & 1.13 & $0.86-1.46$ & 1.63 & $1.46-1.81$ \\
\hline OST $^{\mathbf{a}}$ & 0.59 & $0.52-0.67$ & 0.67 & $0.50-0.91$ & 0.55 & $0.48-0.64$ \\
\hline \multicolumn{7}{|c|}{ Problematic alcohol use $^{a}$} \\
\hline No & I & & 1 & & I & \\
\hline Yes & 1 & $0.90-1.10$ & 0.99 & $0.76-1.28$ & 0.85 & $0.76-0.96$ \\
\hline \multicolumn{7}{|c|}{ Mental health-counseling visits ${ }^{a}$} \\
\hline No & I & & I & & I & \\
\hline 1 & 0.52 & $0.43-0.62$ & 0.53 & $0.35-0.81$ & 0.62 & $0.51-0.76$ \\
\hline$\geq 2$ & 0.46 & $0.4 I-0.52$ & 0.57 & $0.44-0.75$ & 0.40 & $0.35-0.46$ \\
\hline \multicolumn{7}{|l|}{ Active TBa } \\
\hline No & I & & I & & I & \\
\hline Yes & 0.80 & $0.40-1.61$ & 1.48 & $0.20-10.65$ & 0.77 & $0.36-1.62$ \\
\hline \multicolumn{7}{|l|}{ Hepatitis $\mathbf{B}^{\mathbf{a}}$} \\
\hline No & I & & I & & 1 & \\
\hline Yes & 1.34 & $1.16-1.55$ & 1.84 & I.24-2.74 & 1.27 & $1.08-1.48$ \\
\hline \multicolumn{7}{|c|}{ Material deprivation quintile at time of test } \\
\hline QI (most privileged) & I & & I & & I & \\
\hline Q2 & 0.69 & $0.58-0.82$ & 0.67 & $0.44-1.01$ & 0.92 & $0.77-1.11$ \\
\hline Q3 & 0.72 & $0.6 \mathrm{I}-0.86$ & 0.68 & $0.46-1.02$ & 0.81 & $0.67-0.98$ \\
\hline Q4 & 0.85 & $0.73-0.99$ & 0.68 & $0.47-0.99$ & 0.96 & $0.81-1.13$ \\
\hline Q5 (most deprived) & 1.08 & $0.94-1.24$ & 0.73 & $0.5 \mathrm{I}-1.03$ & 1.23 & $1.06-1.44$ \\
\hline Unknown & 1.54 & $1.13-2.11$ & 0.54 & $0.19-1.51$ & 1.89 & I.35-2.63 \\
\hline
\end{tabular}

Notes: Sensitivity analysis using number of mental health-counseling visits per year. aHistory of disease/risk factor in interval between HCV diagnosis and HIV diagnosis. Abbreviations: BC, British Columbia; HCV, hepatitis C virus; HR, hazard ratio; IDU, injection-drug use; MSM, men who have sex with men; OST, opioid-substitution therapy; TB, tuberculosis. 
Table S4 Multivariable Cox regression analysis of factors associated with time to HIV infection in the BC Hepatitis Testers Cohort stratified by major mental illness

\begin{tabular}{|c|c|c|c|c|}
\hline \multirow[t]{3}{*}{ Variables } & \multirow{2}{*}{\multicolumn{2}{|c|}{$\begin{array}{l}\text { Individuals with major } \\
\text { mental illness } \\
\text { HCV infection }\end{array}$}} & \multirow{2}{*}{\multicolumn{2}{|c|}{$\begin{array}{l}\text { Individuals without major } \\
\text { mental illness } \\
\text { HCV infection }\end{array}$}} \\
\hline & & & & \\
\hline & Adjusted HR & $95 \% \mathrm{Cl}$ & Adjusted HR & $95 \% \mathrm{Cl}$ \\
\hline \multicolumn{5}{|l|}{ Age at diagnosis } \\
\hline$<25$ years & 1.52 & $1.06-2.20$ & 2.68 & $2.21-3.25$ \\
\hline $25-34$ years & 1.41 & $1.03-1.92$ & 2.08 & $1.77-2.45$ \\
\hline $35-44$ years & 1.28 & $0.94-1.73$ & 1.65 & $1.4 \mid-1.94$ \\
\hline$\geq 45$ years & I & & 1 & \\
\hline \multicolumn{5}{|l|}{ Year of diagnosis } \\
\hline Before 2000 & $0^{\mathrm{a}}$ & 0 & 1.46 & $0.69-3.09$ \\
\hline 2000-2004 & 0 & 0 & 0.75 & $0.35-1.59$ \\
\hline 2005-2008 & 0 & 0 & 0.69 & $0.32-1.50$ \\
\hline 2009-20II & 0 & 0 & 0.68 & $0.30-1.54$ \\
\hline After 201I & 1 & & 1 & \\
\hline \multicolumn{5}{|l|}{ Urban } \\
\hline No & I & & I & \\
\hline Yes & 1.23 & $0.88-1.72$ & 1.54 & $1.26-1.88$ \\
\hline Unknown & 0.91 & $0.32-2.55$ & 0.70 & $0.39-1.25$ \\
\hline \multicolumn{5}{|c|}{ Sex and sexual orientation } \\
\hline Male, not MSM & I & & 1 & \\
\hline Male, yes MSM & 6.22 & $3.48-11.09$ & 2.41 & $1.80-3.23$ \\
\hline Female & 0.84 & $0.70-1.01$ & 0.78 & $0.70-0.87$ \\
\hline HIV tests per year & 1.70 & $1.62-1.78$ & 1.58 & $1.55-1.61$ \\
\hline \multicolumn{5}{|l|}{ IDU } \\
\hline No & I & & 1 & \\
\hline Yes & 1.96 & $1.5 \mathrm{I}-2.54$ & 1.41 & $1.27-1.58$ \\
\hline OST $^{\mathrm{b}}$ & 0.67 & $0.53-0.84$ & 0.56 & $0.48-0.66$ \\
\hline \multicolumn{5}{|c|}{ Problematic alcohol use ${ }^{b}$} \\
\hline No & 1 & & I & \\
\hline Yes & 1.05 & $0.87-1.26$ & 0.96 & $0.85-1.09$ \\
\hline \multicolumn{5}{|c|}{ Mental health counseling } \\
\hline No & I & & 1 & \\
\hline Yes & 0.39 & $0.31-0.49$ & 0.56 & $0.47-0.67$ \\
\hline \multicolumn{5}{|l|}{ Active TB } \\
\hline No & 1 & & I & \\
\hline Yes & 1.38 & $0.5 I-3.69$ & 0.57 & $0.21-1.52$ \\
\hline \multicolumn{5}{|l|}{ Hepatitis B ${ }^{b}$} \\
\hline No & 1 & & 1 & \\
\hline Yes & 1.32 & $1.03-1.7 \mid$ & 1.33 & $1.11-1.59$ \\
\hline \multicolumn{5}{|c|}{ Material deprivation quintile at time of test } \\
\hline QI (most privileged) & I & & I & \\
\hline Q2 & 0.85 & $0.62-1.16$ & 0.62 & $0.50-0.76$ \\
\hline Q3 & 0.62 & $0.45-0.87$ & 0.77 & $0.64-0.94$ \\
\hline Q4 & 0.74 & $0.54-1.00$ & 0.90 & $0.76-1.08$ \\
\hline Q5 (most deprived) & 0.84 & $0.64-1.11$ & 1.19 & $1.02-1.4$ \\
\hline Unknown & 1.24 & $0.67-2.29$ & 1.68 & $1 .|7-2.4|$ \\
\hline
\end{tabular}

Note: 'Estimates not generated due to lower sample size; 'history of disease/risk factor in interval between HCV diagnosis and HIV diagnosis.

Abbreviations: BC, British Columbia; HCV, hepatitis C virus; HR, hazard ratio; IDU, injection-drug use; MSM, men who have sex with men; OST, opioid-substitution therapy; TB, tuberculosis. 
Table S5 Multivariable Cox regression analysis of factors associated with time to HIV infection in the BC Hepatitis Testers Cohort

\begin{tabular}{|c|c|c|c|c|c|c|}
\hline \multirow[t]{2}{*}{ Variables } & \multicolumn{2}{|l|}{ HCV infection } & \multicolumn{2}{|c|}{$\mathrm{HCV}^{+}$seroconverters } & \multicolumn{2}{|c|}{$\mathrm{HCV}^{+}$-prevalent } \\
\hline & Adjusted HR & $95 \% \mathrm{Cl}$ & Adjusted HR & $95 \% \mathrm{Cl}$ & Adjusted HR & $95 \% \mathrm{Cl}$ \\
\hline \multicolumn{7}{|c|}{ Age at HCV diagnosis } \\
\hline$<25$ years & 2.42 & $1.99-2.95$ & 1.35 & $0.83-2.20$ & 2.04 & $1.62-2.57$ \\
\hline $25-34$ years & 1.80 & $1.51-2.13$ & 0.84 & $0.52-1.36$ & 1.91 & $1.58-2.29$ \\
\hline $35-44$ years & 1.45 & $|.22-1.7|$ & 1.23 & $0.76-2.01$ & 1.25 & $|.04-1.5|$ \\
\hline$\geq 45$ years & I & & 1 & & I & \\
\hline \multicolumn{7}{|c|}{ Year of HCV diagnosis } \\
\hline Before 2000 & 1.24 & $0.39-3.90$ & 3.23 & $0.44-23.6$ & 0.89 & $0.22-3.62$ \\
\hline 2000-2004 & 0.86 & $0.27-2.70$ & 2.57 & $0.35-18.7$ & 0.67 & $0.16-2.73$ \\
\hline $2005-2008$ & 0.83 & $0.26-2.66$ & 1.64 & $0.22-12.1$ & 0.56 & $0.13-2.33$ \\
\hline 2009-20II & 1.03 & $0.3 \mathrm{I}-3.4 \mathrm{I}$ & 1.97 & $0.25-14.9$ & 0.69 & $0.15-3.06$ \\
\hline After $201 \mathrm{I}$ & I & & 1 & & I & \\
\hline \multicolumn{7}{|l|}{ Urban } \\
\hline No & I & & 1 & & I & \\
\hline Yes & 1.31 & $1.08-1.59$ & 1.08 & $0.7 I-I .64$ & 1.39 & I.II-I.74 \\
\hline Unknown & 0.29 & $0.11-0.73$ & 0 & 0 & 0.38 & $0.15-0.97$ \\
\hline \multicolumn{7}{|c|}{ Sex and sexual orientation } \\
\hline Male, not MSM & 1 & & 1 & & I & \\
\hline Male, yes MSM & 1.89 & $1.27-2.83$ & 4.97 & $2.56-9.66$ & 1.43 & $0.85-2.39$ \\
\hline Female & 0.86 & $0.78-0.96$ & 0.97 & $0.77-1.23$ & 0.87 & $0.76-0.97$ \\
\hline HIV tests per year & 1.64 & $1.6 \mathrm{I}-1.67$ & 1.48 & $1.43-1.53$ & 2.27 & $2.20-2.33$ \\
\hline \multicolumn{7}{|l|}{ IDU } \\
\hline No & 1 & & 1 & & I & \\
\hline Yes & 1.67 & $1.48-1.88$ & 1.24 & $0.94-1.63$ & 1.83 & $1.61-2.09$ \\
\hline OST $^{a}$ & 0.71 & $0.62-0.82$ & 0.72 & $0.53-0.97$ & 0.69 & $0.58-0.81$ \\
\hline \multicolumn{7}{|c|}{ Problematic alcohol use $^{a}$} \\
\hline No & $\mathrm{I}$ & & $\mathrm{I}$ & & I & \\
\hline Yes & 1.05 & $0.93-1.18$ & 0.97 & $0.74-1.27$ & 0.85 & $0.74-0.97$ \\
\hline \multicolumn{7}{|c|}{ Mental health counseling } \\
\hline No & I & & 1 & & I & \\
\hline Yes & 0.55 & $0.49-0.62$ & 0.60 & $0.46-0.77$ & 0.50 & $0.44-0.57$ \\
\hline \multicolumn{7}{|l|}{ Active TB ${ }^{a}$} \\
\hline No & I & & I & & I & \\
\hline Yes & 0.40 & $0.13-1.26$ & 1.68 & $0.23-12.15$ & 0.31 & $0.07-1.24$ \\
\hline \multicolumn{7}{|l|}{ Hepatitis Ba } \\
\hline No & I & & 1 & & I & \\
\hline Yes & 1.45 & $|.23-1.7|$ & 1.91 & $1.27-2.87$ & 1.39 & $1.16-1.66$ \\
\hline \multicolumn{7}{|c|}{ Material deprivation quintile at time of test } \\
\hline QI (most privileged) & I & & I & & I & \\
\hline Q2 & 0.73 & $0.59-0.89$ & 0.59 & $0.38-0.926$ & 1.11 & $0.88-1.40$ \\
\hline Q3 & 0.78 & $0.64-0.95$ & 0.69 & $0.46-1.055$ & 0.93 & $0.74-1.17$ \\
\hline Q4 & 0.94 & $0.78-1.13$ & 0.68 & $0.46-1.00$ & 1.13 & $0.91-1.39$ \\
\hline Q5 (most deprived) & 1.09 & $0.92-1.29$ & 0.71 & $0.49-1.01$ & 1.30 & $1.07-1.57$ \\
\hline Unknown & 1.45 & $0.98-2.16$ & 0.59 & $0.21-1.66$ & 1.93 & $1.26-2.97$ \\
\hline
\end{tabular}

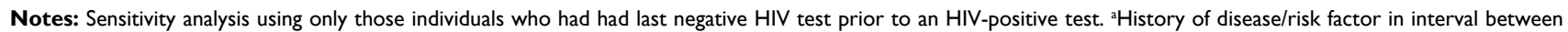
$\mathrm{HCV}$ diagnosis and HIV diagnosis.

Abbreviations: BC, British Columbia; HCV, hepatitis C virus; HR, hazard ratio; IDU, injection-drug use; MSM, men who have sex with men; OST, opioid-substitution therapy; TB, tuberculosis. 


\section{References}

1. British Columbia Ministry of Health [creator] (2014): Discharge Abstract Database (Hospital Separations). British Columbia Ministry of Health [publisher]. Data Extract. MOH (2013). Available from: https://www2. gov.bc.ca/gov/content/health/conducting-health-research-evaluation/ data-access-health-data-central.

2. British Columbia Ministry of Health [creator]. (2014): Medical Services Plan (MSP) Payment Information File. British Columbia Ministry of Health [publisher]. Data Extract. MOH. (2013). Available from: https://www2. gov.bc.ca/gov/content/health/conducting-health-research-evaluation/ data-access-health-data-central.

3. British Columbia Ministry of Health [creator]. (2014): PharmaCare. British Columbia Ministry of Health [publisher]. Data Extract. MOH. (2013). Available from: https://www2.gov.bc.ca/gov/content/health/ conducting-health-research-evaluation/data-access-health-data-central.
4. British Columbia Ministry of Health [creator]. (2014): PharmaNet. British Columbia Ministry of Health [publisher]. Data Extract. MOH. (2013). Available from: https://www2.gov.bc.ca/gov/content/health/ conducting-health-research-evaluation/data-access-health-data-central.

5. BC Vital Statistics Agency [creator] (2014): Vital Statistics Deaths. BC Vital Statistics Agency [publisher]. Data Extract. BC Vital Statistics Agency (2014). Available from: https://www2.gov. bc.ca/gov/content/health/conducting-health-research-evaluation/ data-access-health-data-central.

6. British Columbia Ministry of Health [creator] (2014): Client Roster (Client Registry System/Enterprise Master Patient Index). British Columbia Ministry of Health [publisher]. Data Extract. MOH (2013). Available from: https://www2.gov.bc.ca/gov/content/health/ conducting-health-research-evaluation/data-access-health-data-central.
Clinical Epidemiology

\section{Publish your work in this journal}

Clinical Epidemiology is an international, peer-reviewed, open access, online journal focusing on disease and drug epidemiology, identification of risk factors and screening procedures to develop optimal preventative initiatives and programs. Specific topics include: diagnosis, prognosis, treatment, screening, prevention, risk factor modification,

Submit your manuscript here: https://www.dovepress.com/clinical-epidemiology-journal

\section{Dovepress}

systematic reviews, risk and safety of medical interventions, epidemiology and biostatistical methods, and evaluation of guidelines, translational medicine, health policies and economic evaluations. The manuscript management system is completely online and includes a very quick and fair peer-review system, which is all easy to use. 